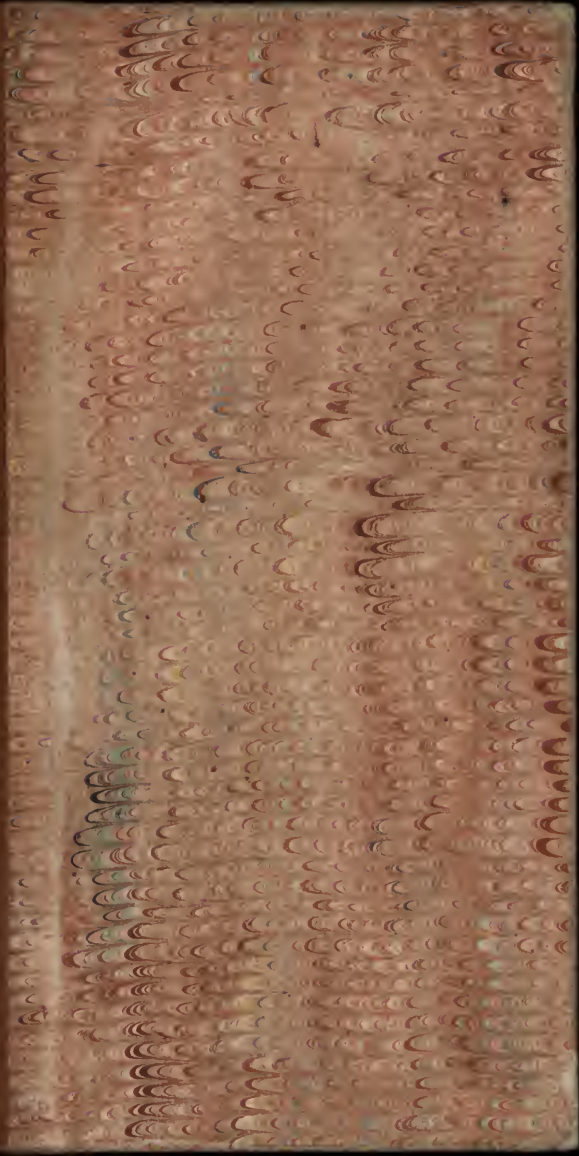




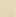

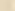




$$
28 A^{41} \text { प }
$$




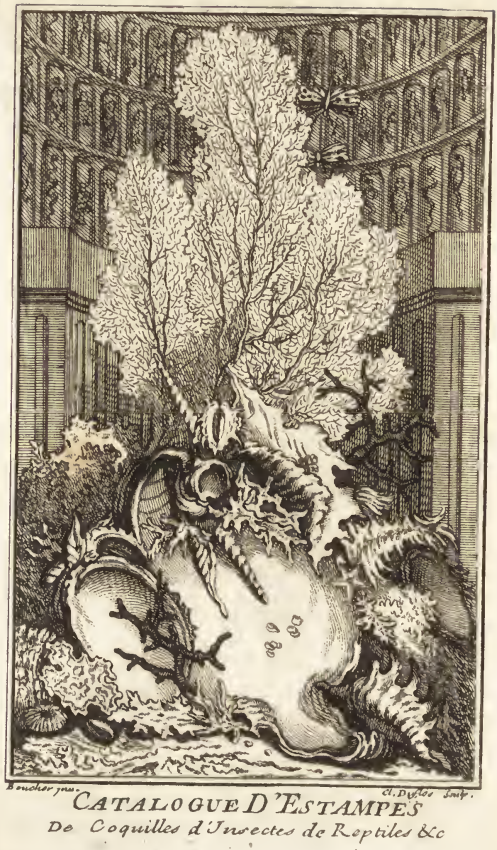




\section{CATAL OGUE}

D'U NE C O L L E C T I O N confidérable de Curiolitez de differens gen res, dont la Vente doit commencer le Lundi 2 Decembre $1 \% 37$. à deux heures après midi, chez Gersaint Marchand Pont NotreDame.

Ces Curiofitez confiftent en un très-grand nombre d'Eftampes d'après les plus grands Maîtres, des mieux conditionnées ; des Deffeins; une grande quantité de Coquillages rares; des Madrepores \& autres Plantes marines; des Animaux dans des phioles; des Mineraux; des Papillons étrangers; de très-belles Pierres arborifées; des Pagodes de diverfes formes, \& autres Ouvrages variés de Porcelaine; des Taffes \& Boëtes d'anciens Vernis;quelques Pierres antiques, montées \& non montées; des Figures en cire très-finies, \& autres Curiofitez \& productions de la Nature \& de l'Art.

\section{Ce Catalogue Se vend}

\section{A PA R I S,}

Chez PR A U I.T fils, Quay de Conty, vï-à-vis la defcente du Pont-neuf, à la Charité.

M D C C X X X V I I.

Ony trouve aufi le Catalogue de la Ventede $\$ 736$. 
On pourra commodémeint voir tout ce gus fait l'objet de cette Vente, à commencer du Lundi 25 Novembre jufgues or compris le $V$ endredi 29 du meme mois, les matins depuis neuf beures jufqu'à midi, or les aprèsdinées depuis deux beures jufqu'à cinq. On engage les Amateurs à y venir pendant le jour, la lumiere n'étant pas également favorable pour en décider, \& surtout par rapport aux Coquillages, qui y perdent leur couleur, de même que les Agathes arborifées, of lés Pierres gravées dont on ne peut pas $f i$ facilement diffinguer le travail. 


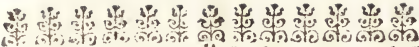

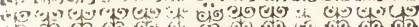

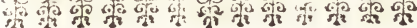

\section{AVERTISSEMENT.}

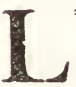

'Idcee que je me fuis formée, a été de ramaffer dans le dernier voyage que je viens de faire en Hollande, une fuite de Curiofitez de divers genres, qui put amufer les Amateurs, \& fatisfaire les gouts differens. Comme j’ai trouvé à mon setour quelques acquifitions a fure, je les y aijontes, \& je crois que lon pouma cere atirć par la varićté \& la fingularité des Moiccaux qui feront expofés à cette Vente.

Le frontifpice de ce Catalogue comprend affez génćralement le total de ce que renferme la Vente, fans ctre obligé de retomber ici dans un nouveau détail que le Catalogue apprendra avec plus de précifion. Un ćloge de toutes ces $\mathrm{Cu}$ riofitez paroitroit fürement fufpect de ma part; \& jaime mieux laiffer au Public la liberté d'en porter lui-même fon jugement.

Je ne puis cependant m'empecher de' ai ij 
dire au fujet de la coliection des Eftampes qui fait une des parties de cette Vente. qu'elle eft le choix de ce qqu'on a pû trouver de plus parfait de chaque Maître, \&r que depuis long-tems il ne s'eft point fait ence genre une vente aufi confidérable, tant pour la quantité, que pour la condition.

Je dois dire ici un mot de la méthode que j'ai fuivie dans larrangement de ces Éftampes : je n'ai paspû être aufî forupuleufement exact dans ce Catalogue, qu'on pourroir l'êtie en faifant le Catalogue d'un Cabinet particulier; $\&$ je ne doute nuilement qu'il ne s'y foit glifié plufieurs fautes. Une parfaite cxactitude \& une précifion irréprochable auroient demandé un tems plus confidćrable, \& méme deviendroient,pour ainfi dire, impoffibles dans un Catalogue de Vente, étant obligé d'opérer autrement que dans un Catalogue particulier, par rapport à la diftribution des articles qui doivent être faits de façon, qu ils puiffent étre propres pour ctre vendus.

Il fuffit, après tout, pour une Vente, de mettre les Curieux en ćtat de voir aifément \& d'un coup d'œil , fi les mor- 
ceaux rares \& les plus recherchés, qui peuvent piquar leur curiofité, ou qui leur manquent dans leur collection, s'y trouvent ; \& c'eft ce que j'ai cu en vûe dans l'ordre que jai fuivi.

Chacun a fa méthode particuliere dans l'arrangement de fa Curiofité. A l'égard des Eftampes, les uns les rangent par Graveurs, \& les autres par Peintres. VoiLì les deux manieres les flus ufitées: la premiere méthode ne peut guéres ćtre d'ufage dans une Vente, parce qu'clle occafionne une divifion dans toutes les fuites qui font gravées par plufieurs Maîtres; \& parce qu'elle obligeroit en ce cas à vendre ces fuites tronquées. Il a donc fallu m'en tenir à la feconde méthode, \& je la trouve beaucoup plus aifée à fuivre, même pour un Curieux.

J'ai divifé le tout en trois Ecoles: l'Italienne, la Flamande, \& la Françoife. J'ai compris dans la Flamande les Maitres Allemans, Anglois, Hollandois, \& généralement tous les Maîtres ćtrangers aux deux autres Ecoles:j'ai fuivi, pour plus grande facilité un ordre alphabetique à chaque Ecole; de forte que le Curieux peut aif́ment voir ce qu'il y a de chaque a iij 
Maitre, en le cherchant dans fon Ecole à fon rang; \& j’y ai fuécifié les Morceaux les plus rares \& les plus recherchés, autant qu'un Catalogue de vente peut le permettre. Gilles Sadeler, par exemple, quoique Graveur Flamand, fe trouve en partie dans l'Ecole Italienne, parce qu'il a gravé plufieurs morceaux d'après le Baifan, qui eft un Maitre Italien : ainf ces Morceaux fe trouveront dans l'article du Baffan, \& non pas dans Particle des Sadelers. Mirs Drevets ont beancoup gravé d'après M. Rigault ; par conf́'quent leur article fe trouvera foible, n'y ayant que les Morceaux quis'y font rencontrés doutbles, \& la plupart de leurs Ouvrages fcront placés fous le nom de $M$. Rigault, \& ainfi des autres Graveurs.

J'ai tâché de ne point féparer dans l'article de chaque Peintre les Pieces gravées par un méme Graveur, afin que ceux qui s'attachent plus au Graveur qu'au Peintre, puiffent fe fatisfaire plus facilement, fans être obligés de fe charger de morcenux qui ne leur conviendroient pas.

Si j’ai trouvé dans un Maitre quelques Pieces répétées, j’ai fait en forte d'en placer une fous le nom du Peintre, \& j'ai ren- 
voyé l'autre à l'article du Graveur.

Je n'ai point divifé les productions des Graveurs qui ont beaucoup travaillć d'aprc̀s eux-mêmes, quoiqu'ils ayent fait plufieurs Morceaux d'après d'autres Maitres, comme Callot, Picard, le Clerc, \&c. J'ai fait la même chofe à l'égard des Graveurs qui font encore plus connus par leurs noms propres, que parles Peintres d'après lefquels ils ont gravé, comme Smith, \&cc.

Les Maîtres qui ne m'ont point fourni affez de Pieces pour faire un article raifonnable pour la Vente, ont été placés avec d'autres à la fin de l'Ecole qui leur convient, fous le titre de Differens Maîtres de cette Ecole.

A l'égard des grandes Suites ou Morceaux particuliers, qui n'ont pas pû fe ranger aifément fous des noms de Mầtres, ou qui font des articles feuls, je les ai mis pour la plûpart enfemble, à la fin de toutes les Ecoles, fous les titres de Galleries, Vûes, Hiftoire naturelle, Arcbitciture, Fêtes, Catafalques, \&oc.

Je crois avoir difpofé les numeros de façon qu'ils puiffent convenir à tous les acquereurs, fans Étre ni trop forts ni trop. foibles,pour la quantité des Morceaux qui 
les compofent; excepté les Pieces rares \& de prix, qui feront vendues feule à feule, deux à deux, \&ic. On a exigé de moi cette méthode, tant par rapport aux Eftampes, que par rapport aux autres $\mathrm{Cu}-$ siofitez.

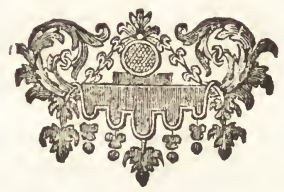

ESTAMPES 


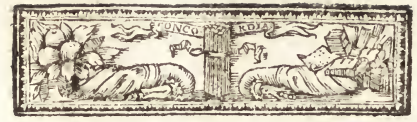

\section{E S T A M P E S}

Rangées par Ecoles \& par Maîtres dans un ordre alphaliélique.

\section{ECOLE ITALIENNE.}

俩 I. 1 veurs, Sculpteurs, Architectes \& *ig Deffinateurs, rangés par ECOLE, 2.

Premier volume, Ecole Italienne; 166 Portraits.

Second volume, Ecole Flamande, $\{367$ Portraits.

Troifiéme volume, Ecole Françoise: ( 63 Portraits.

Faifant le tout enfemble 69: Portraits.

Sous le même No. la vie des Peintres par $V_{a}$ fari, 2. vol. qui peuvent fervir de fupplément à l'article ci-deffus.

Il feroit inurile de vouloir s'étendre fur le mérite d'une collection fi nombreufe : on comprend aifément que ce n'eft qu'avec bien des recherches, des foins \& de la dépenfe que l'on peut parvenir à la conduire à ce point ; le $\mathrm{Cu}-$ rieux quil'avoit formé a pouffé le fcrupule jufqu'à vouloir y mettre, autant qu'il a pû, les différentes fois que chaque Maître a été gravé, craignant (s'il en avoit voulu fouftraire quelqu'un) de rejetter le plus reffemblant, fouvent 
le plus mal gravé étant le meilleur pour la reffemblance, \& l'on a été obligé de rompre quantité de volumes pour avoir de certains Portraits qui ne fe trouvent point gravés autre part. On y a joint le Portrait des Protecteurs $\&$ des Amateurs de cet Art ; cela s'eft obfervé plus facilement dans l'Ecole Françoife, \& on y a compris généralement tout ce qui ef marqué dans la lifte des Portraits de l'A cadémie RoyaJe de Peinture \& de Sculpture.

\section{A L B A N E.}

* Les quatre grands Albanes gravés par Baudet, belles épreuves.

3 Les quatre Elemens par le même, avec deux autres pieces.

\section{B A R O C H E.}

4. Six morceaux, dont trois font gravés par luimême.

$$
\text { B A S S A N. }
$$

5 Seize pieces, dont quinze gravées par Sadeler, toutes parfaites épreuves, \& la feiziéme gravée par Pietre de Jode. C'eft une des fuites des plus piquantes de cette collection.

$$
\text { B E L L E (la) }
$$

Renvoié an $N^{\circ} .83$. dernier article de l'Ecole Italienne.

$$
\text { B E N E D E T E. }
$$

6 Trente-fix morceaux, tant grands que petits; gravés pour la plûpart par lui-meme.

7 Dix autres petits morceaux.

$$
\text { B E R N I N (le) }
$$

8 Six morceaux, dont la Chaire de S. Pierre \& le Crucifis gravés par Spiere, \&c. 


\section{ECOLE ITALIENNE. \\ C A R A C H S (les)}

, Dix grands morceaux gravés par différens Maitres François.

Io Neuf autres, aufi par divers Grayeurs Francois.

II Treize autres par différens Graveurs.

I 2 Trois grandes pieces, fçavoir, la Vierge du Correge, le S. Jerôme du Tintoret, \& la Sainte Juftine de Paul Veronefe, toutes trois belles épreuves \& gravées par Auguftin.

I3 Sept pieces, dont le grand morceau complet d'après le Perugin, gravé par Aug. Car. \& cc.

I4 Douze moiens morceaux gravés par les $\mathrm{Ca}$ raches.

I5 Trente - une petites pieces, Vierges \& autres. I 6 Sept autres pieces : cinq petites femmes \& deur autres plus grandes.

I7 Neuf autres grands morceaux. I 8 Les Cris, gravés par Carache. Ig Les mémes, gravés par Mirelli.

\section{CARLO M A R T TI.}

$20 \mathrm{La}$ Vierge, gravée par Smith, très-belle épreuve.

2I Douze morceaux, dont plufieurs gravés par Frey.

.22 Quinze autres morceaux, gravés tant par luiméme que par d'autres Graveurs.

$$
\text { COR NEILLE CORT. }
$$

23 Trente-cinq morceaux, tant grands que petits:

$$
\text { C ORR E G E. }
$$

24 Douze pieces, par différens Graveurs.

$$
\text { D O M I I Q U I N. }
$$

25 Douze morceaux, gravés par Frey, Gerard Audran, \&x. 


$$
\text { E S P A N O L E T. }
$$

36 Huit pieces, gravées par lui-même.

$$
\text { G E N O I S (le) }
$$

27 Deux morceaux rares, gravés par lui-même:

$$
\text { GEORGES MANTUAN. }
$$

28 La Réfurrection, grande piece, parfaitc \&preuve.

as Neuf autres morceaux différens.

$$
\text { G U ER C H I N. }
$$

3० Quatorze pieces, gravées par différens Maîntres.

$$
\text { G U I D E. }
$$

3I Trente-cinq pieces, tant grandes que petites; gravées par lui-meme.

32 Quarante-cinq autres morceaux, auffi gravés par lui.

33 Sept grands morceaux : les quatre Travaux d'Hercule \& le S. François, par Rouffelet; la fainte Famille, par Poilly, \&c.

$$
\text { L A NFR A N C. }
$$

34 Quatre pieces.

$$
\text { P A L M E. }
$$

35 Onze pieces, par différens Graveurs. PAUL VERONESE.

36 Quatre grands fujets, dont quelques-uns font: en plufieurs pieces.

37 Neuf autres, par divers Graveurs.

$$
\text { PIETRE DE CORTONE. }
$$

38 Les quatre pieces de la Sainte Bibiane, \& la 
Vierge gravée par Spiere, belles ćpreuves. 39 Cinq grands morceaux, par Aquila. 40 Treize morceaux differens, par divers Graveurs.

4I Treize autres, idem.

\section{PIETRE TESTE.}

42 Vingt pieces, tant grandes que petites:

\section{R A P H A E L.}

43 La fainte Famille gravée par Edelinck, premiere épreuve avant les armes.

44 Quatorze pieces: le S. Michel par Edelinck; quelques Vierges, \&c.

45 Les deux belles Vierges, "gravées par Poilly, des premieres épreuves; \& la Transfiguration, par Dorigny.

46 Six grandes pieces: l'Ecole d'Athenes \& le Triomphe du S. Sacrement, par Georges Mantuan; la Nativité, par Bloemart; une grande Bataille, \&c.

47 La Pfiché, gravée par Marc-Antoine en trente-deux morceaux, dont vingt font avant le nom de Salamanque. 48 Dix pieces, gravées par Marc-Antoine. 49 Les A pôtres en treize pieces, aufli par MarcAntoine.

50 Vingt-neuf morceaux, par le méme. $5 \mathrm{I}$ Vingt autres dit.

52 Dix autres, par le même, dont la Sainte $\mathrm{Ce}$ cile avec le collier, très-belle épreuve, \&c.

${ }_{3} 3$ Sept grands morceaux, auffi par M. Antoine. 54 Le Jugement de Paris \& un Triomphe, par le même.

$5.5 \mathrm{La}$ Galathée, du même, premiere '́preuve. 56 Deux différenres épreuves du martyre de Sainte Julienne, par le même, \& dont l'une eft fans l'oreille.

$$
\text { A iij. }
$$


57 Deux maffacres des Innocens, par le même; celui au chicot $\&$ l'autre.

58 Le Parnaffe, par le mème.

59 Le Saint Laurent d'après Baccio Bandinelli, par le même.

60 Quatre grandes pieces, par le même, dont le Neptune, la Carcaffe, \&c.

6I Quinze pieces, gravées par différens Maîtres. 62 Dix-huit autres pieces, idem.

\section{SALVATOR ROSE.}

${ }_{63}$ Dix grands morceaux, gravés par lui-mème. 64 Soixante \& dix autres pieces, dont les Soldats $\&$ trois autres grands morceaux.

\section{T I N T OR E T.}

65 Cinq pieces, dont le Crucifix en trois morceaux, gravé par Aug. Carache, \&c.

\section{T I T I E N.}

66 Les Amours des Dieux, gravés par Smith, en neuf morceaux fansle titre, belles épreuves. 67. Les Difciples d'Emaüs, par Maffon, épreuve parfuite ; le S. Laurent, par Corneille Cort, \& une Vierge, par Blomart.

68 Huit morceaux, tant Portraits que fujets; dont cinq gravés par Hollard.

69 Seize autres, gravés par différens Maîtres.

$$
\text { V A L E N T I N. }
$$

70 Six pieces, dont les quatre Evangeliftes du Cabinet, \&r.

\section{VIL L A M ENE.}

7 I Trente \& un morceaux, dont les Gourmeurs; \&.c.

72 Vingt \& un autres morceaux. 


\section{CLAIR OBSCUR.}

73 'Trente morceaux, grands \& petits, de différens Maitres.

$74 \mathrm{Vingt}$-quatre autres, idem.

75 Quarante autres, idem.

DIFFERENS MAISTRES ITALIENS.

76 Quatorze pieces, tant d'Eneas Vicus que de Jules Bonafone.

77 Quatorze autres, de Baptifta Franco.

78 Dix-fept autres, tant d'Auguftin Venitien, que de Sylveftre de Ravéne.

79 Quarante-cinq morceaux, fçavoir, vingtdeux Portraits de diffërens Maitres, \& trentetrois morceaux de la Pfiché.

80 Soixante morceaux divers.

8 I Trente-fix autres, idem.

82 Dix grands niorceaux, dont cing gravés par Frey, \& fept par Dorigny; le tout d'après des l'eintres Modernes Italiens.

B E L L E (la)

CeMaitre ayant été oublté dans fon rang, on a été oblizé de le mettre à la fin de l'Ecole Italienne.

83 Soixante \& dix pieces de grifonnement.

84 Soixante $\&$ dix-huit pieces en plufieurs livres de Vûes, Caprices, Saifons, Exercices de Cavalerie, \&c.

85 Tente-fix pieces : divers Cartouches \& Frifes. 86 Trente-neuf morceaux : quelques Titres rares, le Livre à deffiner \& plufieurs Tctes.

87 Un Livre de Païfages en douze feuilles, dédié a M. le Di:c d'Anguien : un autre de fix feuilles d'Exercices de Guerre, dédié à M. de Maulevrier, \& le Jeu de Cartes de la Géographic. 88 Trente Paifages grands \& petits. 
89 Vingt-fix pieces: Chaffes, Oifeaux \& autres fujets, dont il y en a vingt-cinq de chez le. Grand Duc.

90 Vingt-deux morceaux : les Polcnois, plufieurs Vierges \& autres fujets, parmi lefquels il y en a dix de chez le Grand Duc.

9I Dix-huit pieces de différens fujets de Theatre, Caroufels, Catafalques, \&c. dont la plus grande partie eft du Grand Duc.

92 Neuf grandes pieces, Vûes de Florence, \&c.

93 Le Pont-Neuf, le Repofoir, avec fix pieces da Cabinet du Grand Duc, \& le Siége d'Arras.

Fin de l'Ecole Italienne. 


\section{ECOLE F L A A N D.}

\section{A L BER T D URER.}

Son Oeurre en Taille-Douce.

$$
S \text { ÇA VOIR, }
$$

94 Ix-huit pieces : fix Portraits, la TEte de Mort, le Cocq \& autres fujets.

95 La Paffion en quinze morceaux, \& $t$ :ois Ecce Homo.

96 Vingt-fept pieces: les Apôtres, les Saints \& autres fujets.

97 Dix-neufpieces: lesVierges \& trois Nativitez différentes.

98 Adam \& Eve, les quatre Femmes nues, le S. Jerôme \& la Mćlancolie.

99 Ie Cheval à la Mort, les deux autres Chevaux, \& deux autres pieces avec leurs contr'épreuves.

Ico Ia Pandore, \& une autre grande piece. Ior Le S. Hubert, \& le grand S. Jerôme.

102 Une Paffion en vingt \& un morceaux, une tête de Chrif, \& cinq autres morceaux extrêment rares.

Ces huit articles qui forment l'Oeuvre d'Albert Durer en Taille-Douce, à peu de chofe près, feront vendus en total, s'il fe trouve des enchériffeurs. On n'en impofe point en difant que cette Oeuvre eft une des plus belles qui fe puife trouver, tout $y$ eft parfait \& conditionné, \& elle a été faite fur un grand nombre d'autres, aiant toujours rebuté les pieces qui ne fe trouvoient pas d'une réputation fans reproche. 
I03 Quarante-trois morceaux choifis dans les Tailles w Doic du méme Maitre.

\section{BER G H E M.}

104 Cinquante-deux morceaux de différente grandeur.

ros Cinquante-deux autres pieces, dont il y en a dix-huit grandes.

I06 Vingt-neuf petites pieces qui fe font trouvkes doubles, \& une grande Danfe de Paifan en hauteur, gravée par Jean Wifçher.

\section{B L O M A T.}

Io7 Soixante \&z dou e pieces, petits livres d'Animaux, de fujets \& de Parfages.

I08 Soixante \& treize morceaux : les Hermites \& autres plus grandes pieces.

\section{B L O E TEII N G.}

rog S. Pierre \& S. Paul en maniere noire, parfaites épreuves.

1 Io Six beaux Portraits d'Amiraux.

II Trois autres, idem.

I I Vingt-deux pieces, tant Portaits, Tćtes que fujets en maniere noire \& autrement.)

II 3 Quatorze autres, idem.

$$
\text { B OLS WERT. }
$$

II $\mathrm{La}$ Vie de $S$. Auguftin en vingi-huit morceaux, avec le reniement de S. Pierre d'áprès Segers, belle épreuve.

$$
\text { CRISPIN DE PAS. }
$$

II Soixante \& douze morceaux.

$$
\text { G H E I N. }
$$

I 6 Vingt-cing pieces, dont le Chien d'après Goltzius, les $\lambda$ potres, \& c. 


\section{G OL T Z IUS.}

i 7 Le Chien, très-belle épreuve.

is 8 Dix grands morceaux : la Galathée, la Vie de la Vierge, \&c.

II 9 Trente-trois morceaux : la petite Paflion, plufieurs Vierges, quelqnes Portraits, \&c. 120 Vingt-trois pieces, dont il y en a fix d'épreuves parfaites.

I2 I Vingt-fept autres: les Soldats, les Sybilles, les Statues, \&c.

\section{G O U D.}

122 L'Oeuvre complette en fept morceaux, parfaites épreuves.

$$
\text { G UI L L E M - B A UR. }
$$

123 L'Oeuvre, édition de 1670 . augmentée de plufieurs morceaux très-rares; le tout faifant trois cens cinquante-cinq pieces.:

\section{G UNS T.}

124 Neuf Portraits.

$$
\text { H O LLAR. }
$$

125 Quinze pieces: le Lievre, le Païfage de Teniers, les différens Manchons, \&cc.

126 Quatre grands morceaux : la Cathedrals d'Anvers, la Madeleine, \&c.

I27 Quarante-deux Païrages d'après le Breugel, \& autres.

I28 Soixante-trois pieces: Animaux, Portraits, Titres de livres \& Charges.

I29 Quarante-trois Portraits.

$$
\text { J OR D A N S. }
$$

I30 Le Roiboit, le Repas de famille, \& les deux Satyres; epreuvesavant le nom de Bloteling. 
I 3 I Les deux Rieurs, la Nativité, S. Martin de Tours, \& le martyre d'une Sainte, toutes bellts épreuves.

I 32 Sept autres pieces, différens fujets.

r 33 Sept autres, idem.

\section{A IR E S E.}

13+ Soixante \& douze pieces, tant grandes que petites.

\section{MA NIERE NOIRE.}

35 Cinq morceaux de Verkolje, dont deux font très-rares; \& cinq autres de Bouys, parmi lefquels fe trouve le Portrait de Marais.

匹 6 Vingt pieces de différens Maitres, tant Portraits que fujets.

337 Vingt-quatre autres, idem.

$x_{3} 8$ Vingt-quatre autres, idem.

139 Vingt-quatre autres, idem.

I 40 Vingt-quatre Portraits, idem.

I4I Vingt-quatre autres, tant Portraits que Sujets.

\section{MATHAN.}

$\mathrm{r}_{4} 2$ Vingt-cing morceaux: les Amours des Dieux, belles épreuves, \&c.

\section{I R E V E L D.}

143 Neuf Portaits.

\section{U L L E R.}

I 44 Le Groupe vû de trois cótez en trois pieces, très-belles épreuves.

I 45 Onze pieces; différens fujets.I

146 Neuf autres pieces, tant Portraits que fujets.

147 Six grandes pieces. 


\section{ECOLE FLAMANDE. \\ NICOLAS DE BRUYN.}

¿48 Seize grandes Pieces: l'A ge d'Or, \&c。

$$
\text { OS TA D E. }
$$

\49 Cinquante morceaux de différente grandeur: PETITS MAITRES.

Tout ce qui fe trouve dans ces petits Maîtres, eft très-beau d'épreuve, \& il y a des pieces très-rares. On fçait la difficulté d'avoir ces morceaux conditionnés, pures \& fans être retouchés, par rapport à leur ancienneté.

$$
\text { LUCAS DE LEYDE. }
$$

¿so La Danfe de la Madelaine, \& le crucifie: ment.

I5 5 La Chafte Sufanne avec un autre morceaus: I5 2 Vingt-fix morceaux. La Paffion, les Apố tres, \& une autre piece.

153 Trente-cinq autres pieces.

$$
\text { A IDEGRAEF. }
$$

\$54 Cent quatre morceaux différens, anciennes épreuves.

$$
\text { GEORGES PENTZ. }
$$

Is 5 Cinquante-neuf morceaux parfaits d'épreut: ve.

$$
H \text { I } S \text { B I N } s \text {. }
$$

I56 Cinquante-quatre pieces.

- HANS SEBADUS BESANi":

it57 Trente pieces.

$$
\text { JACQUES BINCk. }
$$

is 8 Trente-neuf pieces. 
LEONARDGAULTIER, JEAN OU HANS BROSAMER, MARTIN SEHON, \& ETIEN E DEIAULNE.

z59 Quatre-vingt-dix pieces de ces Maitres. MAITRES INCONNUS.

160 Cent fept Pieces.

\section{R I M B R A N T.}

761 Le Portrait de Sylvius \& celui du Maitre Ecrivain, tous deux parfaits d'épreuve. I62 Six pieces de différente grandeur. 163 Vingt-une pieces, idem. 164 Vingt-huit autres, idem. I65 Trente-deux, idem.

I66 Quarante-une, idem. 167 Vingt-huit, idem. 168 Trente, idem.

569 Quatre grands morceaux; la Piece de Cens Frans, la Defcente de Croix, l'Ecce Homo, \&c.

r70 Seize pieces qui fe font trouvées doubles.

\section{RUBENS.}

Tout ce qui fe trouve ici de ce Maître eft parfait pour les épreuves, \& l'on pourroit appeller cette fuite une petite œuvre; du moins tout le beau s'y trouve.

a7 I Sept Grandes Chafles par différens Gra veurs.

I 72 Les dix-huit Payfages.

73 Douze morceaux; le Grand Serpent d'A i. rain, un Crucifiment, le Mariage de S. Jofeph \& de la Vierge, les trois Graces, \&c. ₹74 La Gallerie de Luxembourg en vingt-cinq morceaux, toutes des premieres épreuves.

6 . s La Cene, la Réfurrection du Lazare, la Vi: 
fitation, \& la Chute des Anges; toutes qnatre en hatuteur, \& gravées par Bolfwert \& Soutman.

I76 Onze Portraits, tant grands que petits.

177 Soixante-neuf pieces. tant grandes que petites.

I78 Quatorze pieces, idem.

179 Dix autres grands beaux Sujets: Herodias qui apporte la tête de S. Jean ; S. Ignace guériffant les malades; S. François Xavier, prêchant aux Indes; la Defcente de Croix par Lucas Vofterman; le Martyr de S. Thomas; N. S. crucifié, avec deux Larrons; la Converfion de S. Paul, \&c.

Y80 Dix autres grands Sujets.

181 Dix autres; grands morceaux : trois différentes Affomptions; un Ecce Homo; le Martyr d'un Saint, \&.c.

I8 2 La Préfentation au Temple; la Bataille des Amazones; l'Elevation de Croix en trois feuilles; la Chute des Anges \& l'Adoration des Rois, tous grands morceaux parfaits d'épreuves.

\section{RUGENDAS.}

T83 Seize Sujets de Batailles en maniere noire. 284 Douze autres pieces, idem, fur l'art do monter à cheval.

$$
\text { SA DELERS. (Les) }
$$

185 Vingt-huit pieces, tant Sujets que Payfages: 186 Dix grandes pieces, tant Sujets que Portraits. I87 Vingt-cinq Vierges différentes. I 88 Vingt-cinq Portraits, grands \& petits: I 89 Quatre-vingt-quatorze pieces des Saints de Baviere.

190 Irente-un Payfages de différente grandeur. B ij 
. 91 Les douze Empereurs \& les douze Imperatrices.

192 Quinze morceaux quife font trouyés dozt bles.

$$
S A E R \text { D A M. }
$$

93. Quatorze pieces: les Planetes \& autres $S u-$ jets gracieux, tous belles épreuves.

394 Le Bain de Diane; la Folie; les fix morceaux de l'Hiftoire d'Adam; les cinq Sens, \& les quatre Ages.

I95 Dix-neuf Sujets différens, \& un Bas Relie! en fept morceaux.

I96 Six grands beaux morceaux.

197 Les Vierges Sages \& les Vierges Folles, en cinq pieces, avec un autre Sujet.

\section{$S$ M I T H.}

98 La Madelaine à la lampe, \& celle au chardon, parfaites épreuves.

I99 Le Tarquin; la Pfiché; la Vierge du Baroche \& celle du Correge, tous morceaux rares. 200 Le Bain de Diane; la Venus du Correge, \& deux autres morceaux auffi fort rares.

2 or Six Portraits de. jeunes enfans.

202 Dix Portraits; entr'autres, le Maréchal Schomberg, \&ce.

203 Dix autres Portraits.

204 Dix autres : le Comédien ou Frere Quêteur, \&c.

205 Huit autres Portraits.

2.06 Huit autres: la Conteffe de Salisbury ou la Veuve, \&c.

207 Huit autres, idem.

$$
S \text { U X D E R H E F. }
$$

208 Dix beaux Portraits.

209 Sept pieces tant Sujets que Portraits, entr'autres la Paix de Munfter, ancienne épreuver 
ECOLE FLA M A DE.

* épreuve, avec fa contr'épreuve \& les Bourgmeftres de Hollande, tres-belle épreuve.

T H EODORE DE BR Y.

2 I I Treize piece: tous les morceaux rares y font:

$$
\text { V A N D Y C K. }
$$

Ce qu'il y a de ce Maître eft au plus beau pour les épreuves, \& entr'autres il s'y trouve une collection de Portraits des plus amples \& des mieux conditionnés, puifqu'il $y$ en a deux cent quarante-un, fans y comprendre les doubles qui font un article à part ; prefque tous ceux qui doivent avoir le nomt de Vanden Enden y font de cette façon; \& on a cru bien faire de mettre en un feul article tous ceux qui font à peu près de cette grandeur \& qui paroiffent ne point devoir être féparés, ce qui fait le premier article:de ce Maitre.

$$
\text { PORTRAITS. }
$$

2 I 2 Cent foixante-dix-huit Portraits de moyenne grandeur, la plus grande partie de l'impreflion de Vanden Enden.

213 V ingt - huit autres Portraits de différents: grandeur.

2I 4 . Neuf autres grands Portraits.

2.15 Les Comtes \& Comteffes en treize pięces, \& les deux grand's Portraits de Charles pretmier \& de Cromwel.

2.16 Les dix grands Portraits gravés par Gunft:. 2:i 7 Cinquante - un autres Portraits qui fe fonst trouvés doubles. -

$$
\text { SU JET S. . }
$$

218 La Defcente de Croix par Lueas Vofter: rasa, épreuve extraordinaire. 
2 Ig Le Couronnement d'ćpines par Bollwert: avec fa contr'épreuve.

220 . Six grandes autres pieces: le grand Crucifix, une belle Vierge \& un Silene par Bolfwert, \&c.

221 Dix autres morceaux.

222 Sept autres pieces: le grand Crucifix, \& la grande $V$ ierge aux anges par Bolfwert, $\& \mathrm{c}_{0}$.

$$
\text { V A U V R E M E N S. }
$$

223 Huit morceaux par Wifcher, toutes épreuves parfaites.

224 Cinq autres gravés par Moyreau.

$$
\text { W I E R. I X. }
$$

225 Quarante pieces avec l'âge d'or \& le maria-. ge de Rebecca de Théodore de Bry.

$$
\text { W IS CHER. }
$$

$226 \mathrm{La}$ Fricaffeufe, premiere épreuve avant le: nom de Clement de Jonghe.

${ }_{227} \mathrm{La}$ même auffi fans le nom de Clement: Jonghe.

228 LeVielleux, parfait d'épreuve \& très-difficile à trouver beau.

229 Le Couronnement de la Reine de Suede, piece extêmement rare.

230 Les trois Portraits rares à grande barbe, premieres épreuves.

23 I La Bohemienne \& la mort aux rats, auffi. très-belles.

232 Six grands morceaux : la bataille. des $\mathrm{Hu}$ zards, le coup de piftolet, le four, \&c. Toutes piéces affez rares.

233 Six autres fujets.

234 Onze piéces, tant Portraits que Sujets parmi lefquels il y en a de fort raresa

235 Huit pieces d'après Oftade. 
ECOLE FLAMANDE.

236 Six morceaux : les deux grands Baffans, très-belles épreuves, \&c.

237. Dix-fept morceaux, tant Sujets que Portraits \& Payfages.

DIFEERENS MAITRES

DE CETTE ECOLE.

Dont les ouvrages ne font pas en affez grand noinbre pour avoir pâ être placés dans l'or: dre alphabétique.

239 Quatre-vingt quatre piéces: Sujets, Paifages, \&zc.

239 Soixante \& fept Portraits:

240 La Paix de Munfter, avec cent quatro vingt dix-huit Portraits des députés des différensRoyaumes qui fe font trouvés à la ratification de cette Paix.

24 I Huit grands morceaux \& douze plus perits qui repréfentent des Chaffes de diversanimaux : cette fuite eft très-curieufe, rare \&. fort bien gravée.

142 Treize preces, tant Portraits que Sujets; \& vues, par différens Maîtres, imprimées en. couleurs; morceaux finguliers \& peu com muas.

Fin de l'Ecole. Flamande. 


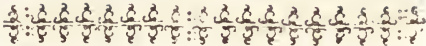

ECOLE FRA NĢOISE. B O U.L O G N E.

243 Ix grandes pieces \& deux petites : la pré$S$ fentation au. Temple par Drevet, \&c.

\section{B O U R D O N.}

244 Seize pieces :les fept œuvres de miféricorde, anciennes ćpreuves, \& des Païfages.

2.45. Vingt-neuf autres morceaux grands \& petits : Vierges \& Sujets.

\section{E- B R U N.}

246 La Franche-Comté gravée par Simonneau, prémiere épreuve..

- 247 Lescing grandes Batailles d'Alexandre, de I'impreffion de Goyton, non affemblćes \& parfaites d'épreuves.

248 La Bataille de Conftantin, fon triomphe, \& la fixiéme Bataille d'Alexandre de chez Bernard Picard, qui n'a point eté gravée par Gerard Audran : article qui peut fervir de fuite au précédent:

249. Les fix petites Batailles d'Alexandre gravées par Jean Audran, épreuves des plus belles.

z 50 Cinq pieces grandes \& petites : la défaite de Porus par Gerard Audran, la Madelaine, Le $S$, Jean l'Evangelifte, \&c.

25 I L'enlevement de Croix \& la defcente de:

Croix par Benoift Audran, le Serpent dairain par Mation, \& un Chrift.

252 Dix-neuf autres morceaux de difiérente 
ECOLE FRANC, OISE. grandeur, tant Sujets que Portraits.

\section{A L L O T.}

II feroit difficile de trouver le Callot plus beau pour les épreuves: il s'y trouve des piéces extrêmement rares, j'ai tâché de fpécifier les morceaux autant qu'il m'a été poffible, \& de faire les articles de façon qu'ils puiffent convenir aux acquereurs.

253 Quarante-fix pieces; I'Enfant prodigue, le Nouveau Teftament fans lettre, le petit Porte-Dieu, S. Jean dans l'Inle de Pathmos; \& les Innocens avec leurs différences.

254 Quarante-neuf autres morceaux; la grande Paffion, la petite Paffion, l'autre petite Paffion ou vie de N. S. en ovale, I'Affomption au chérubin três-rare, \& les quatre petits Banquets, toutes épreuves choifies.

255 Vingt-huit morceaux : les Apôtres \& la Nobleffe.

256 Soixante-quinze pieces : les Gueux, Varie figure ou les Bofus, les Balli ou Curucucu, \&c.

257 Les grandes miferes de la guerre en dix-huit morceaux.

258 Vingt-trois pieces: les petites miferes: les exercices de la guerre, \&c.

259 Les caprices de Florence \& de Nancy, colés à côté l'un de l'autre, trìs-belles épretlves.

260 Dix-fept pieces : le Soliman avec le titre rare \& le combat à la Barriere.

26I Vingt-fept pieces; titres \& autres fujets, prefques tous très-rares : la fainte Apocataftafe, les Géographes, la coutume de Lorraine, la petite Vue de Sienne, le S. François à la tulipe, les fept péchez mortels, les trois. Sacrifices, le Rocher, la Vue du Pont-neuf. 
avec fes différences, le frapement du Rocher aufi avec fes différences, \&c.

z6: Les quatre Bohemiens, les Supplices, la

Pandore avec le Jupiter fans foudre, trèsrare à trouver de cette façon; les quatre $\mathrm{Pai}$ fages parfaits, \& la petite Treille.

263 Onze autres pieces : le Benedicite, les Joueurs de Carte, les trois Pantalons, le Portrait de Delorme, la Vierge d'après Paul Farinat, \&c.

264 Dix-fept morceaux, tant grands que petits: le grand Triomphe de la Vierge, avant le nom de Sylveftre, les deux Vû́es de Paris, un Livre de Païfage, \&c.

265 Le Parterre de Nancy, la grande Rue de

Nancy, \& la Chaffe, trois morceaux parfaits d'épreuve.

266 La petite Foire, ou le Jeu de Boule, avant le nom de Callot, très-rare à trouver ainfi, avec l'Evantail, morceau auffi extrêmement rare.

$267 \mathrm{La}$ Tentation, premiere épreuve, avec les trois morceaux du bas du fiege de Breda. $268 \mathrm{La}$ grande Foire de Nancy, magnifique épreuve, avec l'Epitaphe de Callot.

\section{H A M P G N E.}

269 Dix - fept morceaux, tant Portraits que fujets.

\section{CHEREA U.}

270 Neuf Portraits : M. l'Evéque de Montpellier, \&rc.

\section{CLER C ( le )}

On peut dire ici la mĉme chofe que ce quo j'ai déja dit touchant Callot, tout y eft parfait :les belles \& rares Vignettes s'y trouvent, 
\& j’ai rangé cet article à peu près dans le gout de celui de Callot; cependant il ne m'a pas été poffible de fpécifier auffi régulierement tous les petits morceaux rares, parce qu'il s'en trouve un trop grand nombre dans ce cas, \& que cela m'auroit méné trop loin.

271 Cent vingt pieces : les petits Livres de M. lo Duc de Bourgogne, de M. de Belinghan, de M. de Courtenvaux, \& les petites vûes de Paris.

272 Le Labyrinthe de Verfailles en quaranteune pieces.

273 Les petits Poëtes Italiens fans écriture, \& quelques Médailles : foixante-fix pieces.

274 Les Annales de Toulouze: c'eft une fuite extrêmement rare, puifqu'on ne peut l'avoir complete, qu'en facrifiant le Livre.

275 Les trois Vignettes du livre des animaux avec les trois lettres grifes, \& une de ces trois vignettes, double, \& qui eft connue fous le nom de celle qui n'eft point 'tronquée, elle eft différente de l'autre en quelque partie, \& extrêmement rare.

$276 \mathrm{~V}$ ingt-deux pieces, prefque toutes vignetes rares, \& trop longues à fpécifier : il y a entr'autres le S. Auguitin préchant, le Concile, celle de M. l'Abbé Bignon, \&c.

277 Trente autres petits morceaux : les douze vignetes de l'Hiftoire Eccléfiaftiqque; la petite Venus fur les eaux, rare; les quatre Jeux; N. S. qui prêche dans le Défert, très-rare, \&xc. 278 Vingt-cleux petits titres \& culs de lampe.

279 Onze pieces; les quatre de la Pfiché, quelques morceaux du Vitruve, \&c.

280 Les petites Batailles d'Alexandre en fix morceaux, premieres épreuves, connues fous le nom de l'épaule nue : cette différence fe trouve à une figure qui eft affife dans un des coins 
24 E S T A M P S. de la piece de la famille de Darius.

281 L'hiftoire de Bouillon, trente-fept morceaux, parce qu'il y en a quelques-uns doubles, par rapport aux différences. Cette fuite eft finguliere, \& ne fe trouve pas facilement ainfi.

282 Les huit petites Conquêtes; le Siege de Mons, \& le paffage d'Ifai.

283 L'Hiftoire de Lorraine en trente-fix morceaux.

284 L'Academie des Sciences, premiere épreuve; \& l'entrée d'Alexandre, la Rare, connue fous le nom de la Tête Retournée : dans les autres épreuves, la tête d'Alexandre eft vûe de trois quarts; $\&$ dans cetre épreuve elle eft - tout-à-fait tournée, fans que l'on puifte voir le vifage d'Alexandre.

$\$ 85$ Les deux mêmes pieces : l'Alexandre à la tête Toarnée, \& l'Ácadémie avant le Squelet, que l'on trouve ordinairement dans les autres épreluves, attaché au haut d'une des colomnes: cette picce eft des plus rares à trouver ainfi.

286 Sept Apothéofes d'Ifis, avec des différences; y compris, une épreuve imprimée en rouge, \& deux contr'épreuves différentes : article piquant pour ceux qui font l'œuvre, \& peut-étre unique.

$\$ 87$ Vingt-quatre pieces: le May des Gobelins, le rare; la Proceffion des Chevaliers du SaintEfprit avec les Vignetes, \&c.

288 Douze morceaux : le Mariage de Madame de Bourgogne, Ipar Simonneau; huit animaux du Cabinet, non tronqués, \&c.

289 Les Tapifferies du Roy, parfaites d'épreuves; fçavoir, les quatre Saifons, les quatre Elemens, les quatre Conquêtes, \& la RéduCtion de Marfal. 
ECOIE FRA NC,OISE. 25 290 Le Carafalqzue du Roy de Suede avant la lettre; la pierre du Louvre avant l'année, \& l'Arc de Triomphe.

$$
\text { C O Y P E L S. }
$$

291 Douze Sujets, grands \& petits, de métamorphofe, \& autres.

292 L.e Portrait de Mlle le Couvreur; le Puits de Rebecca; l'Annonciation \& le Sacrifice d'Abrahain; quatre grandes pieces, gravées par Drevet.

293 Six grands morceaux : la Sufanne condamnée; Moife fur les eaux; l'A pothéofe d'Her-. cule, \&c. premieres ćpreuves.

294 Treize morceaux; fujets de Métamorphofe; \&c. Y compris le grand Portrait de la Voifin.

\section{R E V ET S.}

295 Le Portrait de M. le Maréchal de Villars;

épreuve parfaite avant la lettre. Il eft rare.

296 Quatorze beaux Portraits d'après différens Maitres.

\section{E D E L I N C K.}

297 La fainte Famille de Raphaël, premiere épreuve avant les armes.

298 Les porțraits de Dilgerus \& de Remigius du Laury, parfaites épreuves.

299 Dix beaux Portraits, avec la grande Bataille d'aptês L. D' la Finfe.

300 Vingt - quatre pieces, grandes \& petires; Vierges, Sujets, Portraits, Vignetes \& $\mathrm{Ti}_{-}-$ tres de Livres.

$$
\text { G I L L O T. }
$$

3or Les quatre Fêtes du Dieu-Pan; le Bacchanal, gravé par Sarrabat; un morceau d'Ornement, \& les petites Fables de la Mothe : le touţ faifant cent dix pieces. 
302 Sept morceaux de différente grandeur.

$$
\text { J O U V ENET. }
$$

303 La Réfurrection du Lazare \& la Pêche, par Jean Audran, d'après les tableaux de Saint Martin.

\section{A R G I L L I E R E.}

304 Six Portraits : celui de Keller en petit, trèsrare; celui de le Brun, \& celui de Charles Gobineau; tous trois par Edelinck, \& les trois autres par Drevet.

305 Huit autres Portraits; Magalotti, Vandremeulen, \&c.

\section{A S O N.}

306 Les Portraits du Duc d'Harcourt, de Charles Patin, \& de Charier, Lieutenant Criminel de Lyon, tous trois des premieres épreuves. 307 Dix autres Portraits.

$$
\text { M E L L A N. }
$$

308 Le S. Pierre Nolafque, extrêmement rare. 309 Trente-huit autres morceaux.

\section{I G N A R D.}

3 10 Vingt-deux Portraits \& Sujets de différente grandeur.

3 II Neuf pieces, tant Portraits que Sujets: 1o grand Portement de Croix, \&c.

3 I2 Sept Portraits : le Duc d'Harcourt ; le Brifacier, \&c. tous gravés par Maffon.

$$
\text { L E M O I N E. }
$$

3r3 Hercule \& Omphale; Andromede; la Bai- 


\section{ECOLE FRANCOISE.}

gileufe; l'Annonciation; Jacob en Méfopotamie; les Chevaliers Danois, \& le Plafond će la Banque; toutes premieres épreuves.

\section{N A N T E U I L.}

314 Vingt - trois Portraits : le Petit Mylord \& l'A vocat de Hollande s'y trouvent. Ce font les plus rares de ce Maître.

\section{O U D R Y.}

3Is Quatorze Pieces.

\section{P I C A R D.}

- Ce qui fe trouve ici de ce Maître eft confidérable, tant pour le nombre que pour la condition, \& la rareté de certains morceaux; \& cornme il a beaucoup travaillé en titres \& rignetes, il ne in'a pas été poffible de les fpécifier. Je puis dire, fans trop a vancer, que c'eft le choix de tout ce qu'il a fait de plus beau ; \& qu'excepté ce qu'il a donné dans les cérémonies Religieufes, il n'y manque rien d'effentiel.

3 I 6 Soixante-cinq pieces: Modes, Tabatieres \& Cornalines.

317 Trente-deux Portraits : entr'autres, ceux du Concile de Conftance.

318 Trente-huit petits titres de Livres.

319 Cinquante-un petits titres de livres, \& autres pieces : le Roffignol; l'Iliade d'Homere, \&c.

320 Trente - un morceaux : Vignetes \& Sujets; \& entr'autres, trois morceaux de la Bible de Mortier, parfaitement beaux $\&$ très-rares. 3 2 I Trente - fept morceaux : grandes \& petites Vignettes, tant in $-4^{\circ}$. qu'in-I 2 . des plus belles, \& quelques Médailles.

322 Quatorze autres morceaux : petits Titres \& 
Vignetes; \& entr'autres un grand morceau qui repréfente le Cabinet d'Hiftoire naturelle de Levinus Vincent à Harlem. Cette piece eft fort rare.

B23 Onze Nóces: la Minerve rare, avec les vers, \& un beau titre in- $4^{2}$. toutes épreuves choifies. Ces Nôces font les fujets les plus galands $\&$ les plus interefrans qu'il ait fait.

324 Le Maffacre des Innocens avec le Triomphe de la Peinture; deux morceaux des plus renommés.

325 Le grand \& le petit Boileau, en quatre-vingtfix pieces.

326 Le Fontenalle en foixante-onze morceaux.

327 Cinq des plus beaux Titres in-folio.

328 Cinq autres, idem.

$3=9$ Huit grand's morceaux, tirés d'un Livre infolio, de Nivelement.

330 Dix morceaux, différens Sujets: la Vénus avec le Satyre, double, avec fes differences, \&c.

33 I Neuf grandes autres Pieces.

332 Renaud \& Armide; Alexandre \& Roxane, avec une grande Bataille d'Alexandre.

933 Le Meleagre en fept pieces, y compris le titre.

334 Huit morceaux qui fe font trouvés doubles: la Minerve; le Triomphe de la Peinture, le Titre du grand Boileau; le petit Titre de la Eontaine, \&c.

\section{P O US S I N.}

335 Sept grands morceaux : fçavoir, fix Sacrecremens, gravés par Pefne; \& le Baptême do S. Jean, par Gerard Audran.

$33^{6}$ Dix autres morceaux, dont il y en a cinq qui font du cabinet du Roy; le frapperment. du Rocher, par Mll: Stella, \&c. 
337 Dix autres grands morceaux; Vierges $\&$ autres Sujets.

\section{R I G A U L T.}

$33^{8}$ Le Portrait de M. Samuel Bernard, par Drevet.

339 Le Portrait de M. Boffuet, Evêque de Meaux, par le même.

340 Le Portrait de M. de Villars, par le même. 34 I Les Portraits du Cardinal du Bois \& de $\mathbf{M}_{\text {. }}$. de Beauvau Evêque, par le même:

342 Cinq Portraits, tous gravés par François Chereau; M. le Card. de Polignac, \&c.

343 Huit autres gravés par Edelinck : le Marêchal de Villeroy, M. d'Hozier, \&c.

344 Madame, Ducheffe d'Orleans, par Simon-

neau; \& Madame de Nemours, par Drevet. 345 Six autres Portraits, dont trois gravés par-

Drevet: Boileau; M. Dodun, \&c. 346 Dix autres, grands \& petits, par Drevet, \& autres Graveurs.

347 Douze autres, idem:

\section{LE S UEUR.}

348 Vingt-fix morceaux : le Martyr de S. Laurent; Alexandre malade; la Vie de S. Bruno, \&c.

T OURNIER E.

349 Quatre Portraits, par Drevet \& Cliereau.

$$
\text { TRO Y ES. (de) }
$$

350 Huit Portraits, grands \& petits, par Edèlinck.

35 I Onze pieces, dont dix Portraits par differens Graveurs; \& la grande Pefte de Marfeidle, par Thomafin..

$$
\text { C iij) }
$$




\section{A N I O.}

352 Cinq morceaux, gravés par Drevet \& Chereau.

$$
\text { VER D I E R. }
$$

353 La Vie de Samfon, par Benoît Audran, en quarante morceaux, compris le titre.

$$
\text { VOUET. }
$$

354 Trente-deux pieces,

$$
\text { W A T T A U. }
$$

355 Dix morceaux par différens graveurs.

356 Dix auties, idem.

357 Trois autres grandes pieces: les plaifrs du $\mathrm{Bal}$, la Mariće du Village \& l'Enfeigne.

\section{DIFFERENS MAITRES FRANCOIS}

Dont it ne s'eft pas trouvé un aflez grand nombre de morceunx pour être rangés felon l'ordre alpbabétique \& former un article.

$$
\text { P O R T R A T S. }
$$

358 Trente grands Portraits par différens Graveurs.

359 Cinquante autres, idem.

360 Quarante-fix autres, idem.

361 Cinquante-huit autres, idem.

362 Le Portait du Roy à cheval par Thomarfin, \& celui de Mlle Dufrefne par l'Epicier.

$$
S \text { U I E T S. }
$$

363 Trente-trois Sujets modernes.

3.64 Dix-fept grands morceaux de diffírens Graveurs.

365 Trente-trois pieces. Les quatre grandes mo- 


\section{ECOLE FRANCOISE. 3I}

des de Saint Jean, quelques morceaux de Boffe, sce.

366 Cent feize petits morceaux : vignetes, culc de lampe, titres de livres, \&c.

367 Un paquet de divers Eftampes. 368 Un autre paquet.

\section{Fin de l'Ecole Francoife.}

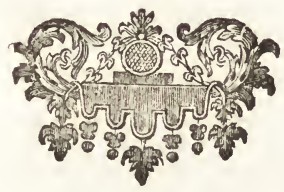




\section{SUITES ET VOLUMES D'ESTAMPES}

\section{de différens Maîtres \&o Ecoles.}

GAIIERIES ET PIAION DS.

369 A Gallerie de Iuxembourg d'après 1 Rubens, en vingt-cinq morceaux.

370 Les angles de Michel Ange par Georges Mantuan : fix pieces.

37 I Un grand plafond de Lanfranc par Cefius en dix pieces.

372 Les douxe Apótres \& les quatres angles de

Lanfranc gravés par Louvemont.

373 L'œuvre complete de le Febvre de Venife.

374 Plafond du Correge en quinze morceaux, par Bonaveri.

375 Meleagre d'après le Brun par Bernard Pi-

card, en fept morceaux, compris le titre.

376 Gallerie de Lanfranc peinte dans le Vatican par Pietro Sancti Bartoli en dix-huit morceaux.

377 Loges de Raphael par Chaperon.

378 Gallerie du Carache peinte dans le Palais

Farnefe, gravée par Cefius.

379 Autre Gallerie du Carache gravée par Mitelli.

380 Autre du même par Mignard d'Avignon. 38I Autre du méme, peinte au Palais Farnefe, gravée par Chatillon.

$3_{82}$ La méme Gallerie gravće par Jacques Belly, épreuves \& contr'épreuves.

383 Gallerie di San Diego par le Carache, \& gravée par Simon Guillain

384 Triomphe de Jules Cefar, Gallerie d'André Manteigne, gravće par Audenaerde de. Gand. 
DIFFERENS MAISTRES.

385 Gallerie du Dominiquain, par le même. 386 Gallerie du Titien, gravée par Mitelli.

387 Plafond de l'Aurore, par Louis Simonneau.

Plafond de la Chapelle de Sceaux, par le même, gravé par Gerard Audran.

388 Trois Plafonds de le Brun : celui du Préfi-

fident Lambert, par Picard; celui du Semi-

naire de S. Sulpice, \& celui de l'Aurore, par

Louis Simonneau, avec une Gallerie, gravée par Saint-André.

389 Gallerie de Pietre de Cortonne, peinte dans le Palais Barberin, gravée par Blomart.

\section{Stites détacbées dil Cabinet du Roy.}

390 Les trente-fix Tableaux du Cabinet du Roy, premieres épreuves, avec le difcours; \& done la famille de Raphaël eft fans les armes. On y a joint quatre autres Eftampes, quí font, la grande Transfiguration de Raphacl; la Vierge du Correge; le S. Auguftin \& le S. Guillaume du Lanfranc, \& la Sufanne du Valentin. Ce qui fait en tout quarante morceaux,

39I Les Conquétes de Louis X I V. completes en trente-huit pieces, gravées par le Clerc, Châtillon, \&ic.

392 La Grotte de Verfailles, avec le Difcours.

393 Les douze Tapifferies du Roy avec les devifes, vignetes \& lettres, gravées par le Clerc, toutes êpreuves parfaites. On y a joint $\mathrm{l} 2$ Réduction de la Ville de Marfal.

\section{Statues, Buftes \& Bas-Reliefs.}

394 Soixante-un Buftes \& Statues de Mellan. 395 Les vingt-une Statues, gravées par Mellan, qui fe trouvent dans la Gallerie Juftinienne. 
396 Trente-huit Statues, gravées par Defplaces, Cochin \& autres; la plûpart d'après les originaux, qui font dans les Jardins du Roy.

$3 \% 7$ Le Volume des Statues antiques de la Gallerie Juftiniane. C'eft le Volume le plus intereffant.

398 Antiques de Perrier, épreuves de Rome, avec les contre-épreuves à côté des ćpreuves. 399 Cent fix Buftes \& Statues antiques du Cabinet de Rince.

400 Bas-Reliefs de Perrier, volume relić.

40I Seize Statues \& Groupes, gravés par Goltzius \& Muller.

402 Soixante-fept morceaux : Bas-Reliefs \& Figures antiques de diffërens Maitres.

403 Le Cabinet de François Boffuit, gravé par Matys Pool, en cent trois morceaux, relié. $404 \mathrm{La}$ Colomne Trajane, gravée par Pietro Santi di Bartoli, reliée.

\section{Architecture, Vues, Bâtimens, Hôtels, Fontaines, \&c.}

405 Vingt-deux grandes Vûes de Silveftre, \& a utres.

406 Cinquante-trois petites V tues de Rome, \& autres lieux, par Sylveftre.

407 Deux Vûes de Marfeille, par Rigault; Elevation, Plan \& Coupe du Louvre, par Marot; Perfpectives de la Ville \& ruines de Bruxelles, en douze pieces, \& autres Vûes \& Elevations, par Marot, \& autres. 408 Théatre de la Grande Bretagne, 4 vol. in-fol. 409 Un Volume oblong relié, contenant cinq Livres, de Ruines \& Bâtimens antiques, Juifs, Fgyptiens, Romains, Arabes, Turcs, Perfans, \& Vafes antiques, Grecs. \& Romains. A Leipfik, 1725 . 
DIFFERENS MAISTRES.

4 Io Trois grands Livres de Vûes, Plans, Elevations de maíon, Hôtels, \&c. gravés en Allemagne.

4 I I Six alitres fuites dans le même goût, auffi gravées en Allemagne. Ces fuites font confidérables \& fort curieufes.

4 I 2 Un autre Volume.d'Architecture, auffi gravé en Allemagne; \& une fuite de Vûes \& Façades, de quelques Hôtels de Vienne.

413 Un gros Volume de Vûes de Rome, par Falda, en quatre Livtes, qui font enfemble cent trente-trois morceaux.

$\int$ Roin $x$ Antiqux \& Nov $x$ Theatrum. $414\left\{\begin{array}{l}\text { Romanorum Fontinalia Norimbergx; } \\ \text { I684. \& 85.2. vol. in-fol. }\end{array}\right.$

415 Les Fontaines de le Brun.

\section{His toire NAtureli.e.}

Et autres fuites qui n'ont pas pû être mijes par articles féparés.

416 Plantes des environs de Paris, parVaillant. in-fol.

4I7 Thefaurus Cochlearum, Concharum, \&c: Rumphii. in-fol. relié.

4 I 8 Animaux de Jonfton; il y manque quelques feuilles.

4 I9 Martini Lifter Hiftorix Conchyliorum, infol. Livre extrêmement rare. Il s'y trouve quelques imperfections; mais il eft très-diffcile de le trouver parfaitement complet.

420 Proverbi Figurati, par Mitelli, in-fol. relié. 42 I Médailles du Regne de Louis XV.

Ce Monde dans une Noix, contenant les événemens les plus remarquables du $422\{$ monde, gravés par Weigel. Les faits mémorables du dix - huitiéme fiécle, gravés par le même. 
36 ESTAMPES. DIFFERENS MAISTRES. 423 Enfans de Siella, reliés.

Maufolés, Catafalques, Décorations, Fêtes, Feux d'Artifices, Eglifes, \& autres Pieces Hifloriques.

424 Trois fuites de Décorations de Théatre, pat Silveftre \& la Belle.

425 Le Caroufel, par Silveftre.

426 Fête donnće à Bruxelles au fujet de l'Empereur Leopold I. gravée en neuf morceaux, par Romain de Hoge.

427 Une fuite de Feux d'Artifices, Entrées \& Fêtes.

428 Le Tombeau de Gregoire XIII. par Frey; celui de M. de Turenne, par Simonneau; \& celui de Girardon.

429 Quarante Tombeaux ou Catafalques.

430 Vûes d'Eglifes, dix pieces.

43 I Trente-fept morceaux, tant grands que petits de différens Sujets allégoriques; parmi lefquels il y en a de rares \& de finguliers.

432 Trente-deux Conquêtes du Roy, gravées par le Clerc, \& autres. Il en manque fix pour être completes.

433 Divers Deffeins, parmi lefquels il s'en trouve de très-beaux, qui feront divifés à la rente en plufieurs lots.

\section{Fin des Eftampes.}


2

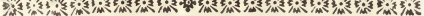

AUTRE AVERTISSEMENT

Touchant ce qui fe trowve d'Hiftoire Naturelle dans ce Catalogue.

Comme l'amour de l'Hiftoire Naturelle ne m'a point paru rallenti, j'ai tâché d'exciter de nouveau les Curieux. qui donnent dans ce genre, par des chofes qui puiffent les piquer, tant par leur rareté, que par leur perfection.

Il fe trouve en tout genre de curiofité, deux Claffes que lon pourroit divifer en Curieux qui fe forment, \& Curieux formés : Les premiers n'ayant pas encore fait apprentiffage de curiofité , s'effarouchent quelquefois du prix d'un morceau rare $\&$ parfait, \& s'en tiennent ordinairement $\mathbf{z}$ ce qui fe trouve plus facilement, \& qui eft d'un prix plus modique; Les feconds au contraire fe livrent plus amoureufement aux Morceaux rares \& parfaits, \& par conféquent plus chers; ayant acquis par l'Habitude une connoiffance plus exacte, ils deviennent plus clair-voyans, $8<$ en même tems plus difficiles dans leur choix; $\&$ comme ils fçavent par une expérience journaliere que le parfaitement beau fe

D 
trouve avec peine, ils ne refufent point d'y mettre le prix convenable pour s'en procurer la poffeflion; j’ai été obligé de fatisfaire ces deux Claffes, les uns \& les autres m’ayant engagé à me fournir de ce qui pourroit leur convenir.

J'ai donc apporté tous mes foins dans ce dernier voyage à faire une collection variée, tant pour les prix que pour les chofes. Je ne m'en fuis pas tenu à la feule partie des Coquillages, $\mathrm{j}$ 'ai acquis des $\mathrm{Pa}$ pillons, des Mineraux, des animaux dans des phioles, des Pierres arborifées, des Madrepores, \& autres Plantes marines, \&c.

Je me fuis trouvé en Hollande heureufement à la fuite d'une Vente fameufe, qui s'eft faite quelque tems avant mon arrivée, \& à une autre Vente qui s'eft faite pendant mon féjour à Amfterdam: ce qui m'a mis en état d'acquérir plus aifément ce que je cherchois; fans cette heureufe conjoncture, j'aurois eu de la peine à trouver de quoi maffortir en cette curiofité, n'étant pas facile à préfent de trouver des Morceaux finguliers \& parfaits chez ceux qui enfaifoient cy-devant commerce. Depuis plufieurs années on ne leur apporte plus rien en ce genre des Indes ni des autres Païs, où ils envoyent des 
vaifleaux, cette partie ne faifant pas in: objet de commerce affez confidérable pour ceux qui reviennent de ces Païs; \& qui plus eft, ces fortes de chofes n'étant rapportćes la pluppart du tems que par des gens qui n'ont nul goût \& nulle connoiffance; ce qui fait que le plus fouvent ils ne fe chargent que de pieces mediocres, communes, fans condition \& fans mérite, $\&$ dont par conféquent its ne peuvent retirer qu'un foible profit, quiles rebute par la fuite, \& les empêche de s'en fournir une autre fois.

Ces deurx Ventes m'ont produit entre attres des Madrepores \& autres Plantes marines d'une beauté finguliere, \& audeffus de toutes celles que j'ai poffédées jufqu'à préfent : ce font des Morceaux qui ne fe trouvent pas ordinairement bien confervés, \& j’en ai vû très-rarement dans les plus fameux Cabinets de la Hollande.

Je ne me fuis point attaché à fpécifier tous les lots de Coquilles qui feront vendus, aufi exactement que dans la vente que je fis au commencement de lannée I 736 . pour laquelle je fis un Catalogue raifonné. Si javois fuivi ce plan, j'aurois fatigué le Public, ou par une lecture ennuyeufe des mémes articles fouvent répétés \& \& dent la férilité de la matiere au-

D ij 


\section{0}

roit pû rebuter, ou enfin par des redites ridicules, $f_{1} j$ 'avois voulu ajouter aux articles les mêmes notes de cet ancien Catalogue.

Cependant s'il fe trouve quelques $\mathrm{Co}-$ quilles affez rares pour mériter d'en faire mention, \& dont je n'aurai point eu occafion de parler il y a deux ans, je ferai en forte de ne les pas paffer légerement, \& fans les faire remarquer.

Je prie ceux qui trouveront quelques articles qui ne leur feront pas affez connus, de vouloir bien avoir recours au Ca. talogue de l'année 1736 . quife vend chez le fieur Prault fils, Quay de Conty; j'efpere que par fa lecture ils fe mettront aifément au fait de certaines Coquilles, \& fe familiariferont en peude tems avec ces noms bizarres qui pourroient les effaroucher. Je faifis icil loccafion de marquer au Public ma reconnoiffance de l'accueil qu'il a bien voulu faire à ce Catalogue dans le tems qu'il parut ; \& je n'ofois pas me flatter qu'un auffi foible Ouvrage eut pû lui être de quelque utilité, \& lui procurer quelque agrément.

Quoiquil ne fe trouve pas un fi grand nombre de numeros qu'il y en avoit dans l'ancien Catalogue, \& que je ne me ferve pas fi fouvent de l'ćpithéte de rareou 
de parfait, on ne doit pas pour cela en tirer la conféquence, que les Coquilles en foient moindres en quantité , ou inférieures en qualité. J'ai.cherché autant que j'ai pû à faire un Catalogue court, \& à y éviter les exclamations répétées, aufquelles on n'ajoute pas toujours foi, quand elles. partent d'une main intereffée. J'ofe dire cependant, que le coup d'œil que l'on peut s'en procurer en les voyant, fatisfera infiniment, \& que l'on y verra nombre de ces jeux admirables de la Nature, \& de ces Coquilles merveilleufes qui tombent rarement fous la main.

Outre les articles de Coquilles qui font fpécifićes dans ce Catalogue, j’en pofféde une fuite auffr complete \& auffi conditionnée qu'on puiffe la faire : elle eft totalement variée dans les efpeces, \& aucun Morceaư n'y eft répété, à moins qu'il ne. $s^{\prime} y$ trouve quelque difference affez fenfi-ble pour.le faire fouhaiter. J'avois commencé cette collection il y a déja dutems, \& je fuis parvenu cette année à la pouffer: it un point affez parfait pour qu'elle puiffe contenter un Curieux difficile $\&$ délicat; $;$ qui voudroit en un inftant fe procurer ce: qu'il ne pourroit acquérir qu'avec beart:coup de foins, de travail, de tems \& dê : dépenfe. On fent bien qu'une colléctiors 


\section{2}

aufli variće \& aufli parfaite ne s'eft pû faire que par la facilité de pouvoir puifer dans un aulfi grand nombre qu'il m'en a paffé par les mains. S'il fe préfente quel. que Curieux pour cette fuite, je lui vendrai à l'amiable, 8 je ne l'expoferai point à cette Vente.

Comme il eft impoffible de donner une idee jufte de ce que repréfente une Pierre arborifée, j’ai crû faire plaifir au Public d'en faire graver une quinzaine que je pofféde, \& que je puis qualifier hardiment de belles \& fingulieres; je les ai fait graver telles que la Nature les a ornćes, fans aucun fard, \& de la même grandeur que la Pierre, afin que l'on en puifie mieux juger: on pourra quand on le voudra, reconfronter les copies à côté des. originaux, je ferai toujours prêt à donner cette fatisfaction; on en trouvera la planche au folio $6 \mathrm{I}$. de ce Catalogue à côté de b'article des.Pierres arborifées.

Je crois que les Curieux auront occafion de fe fatisfaire autant dans les autres: genres de curiofité que comprend cette Vente, comme les pierres gravées, les. Porcelaines, \&c. que dans cette partic de 'Hiftoire naturelle, \& qu'ils pourront s' $y$ ' armufer également. 


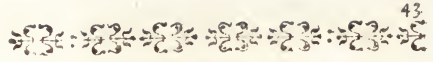 \\ HISTOIRE NATURELLE,
}

\section{ET AUTRES CURIOSITEZ.}

\author{
Premier Tirotr du Coquillier.
}

434 $C E$ Tiroir eft rempli de diverfes Bivalves, qui feront mifes à la vente en plufieurs lots.

\section{TIROIR.}

4.35 Diverfes Coquilles. La plupart, Porcelaines, qui feront également détaillées.

\section{I I. TIROIR.}

436 Deux Choux; une Huitre, \& un grand Manteau Ducal.

437 Deux Coeurs de Venus très-beaux, \& uneHuitre épineufe.

$43^{8}$ Deux grofles Noix de mer, \& deux groffes Fraizes.

439 Un grand Cour de Venus, \& un Manteati: Ducal.

440 Deux petits Cours de Venus; deux autres

Cours triangulaires, \& une Huitre épineufe. 44I Deux grandes Concha Veneris.

442 Un grand Chou, \& deux Cœurs de Bœuf différens.

443 Deux petits Choux, \& une Concha Vineris. 444 Le reftant du Tiroir qui fera divifé à la vente.

$$
\text { I V. T I ROIR. }
$$

${ }_{445} \mathrm{Ce}$ Tiroir eft rempli de perites Coquilles, tant Bivalves qu'Univalves, toutes parfaites; parmi lefquelles il s'en trouve de fort rares, \& dont le détail auroit été trop long. Elles 

feront partagées en différens lots.

$$
\text { V. TI R O I R. }
$$

446 Coquilles Univalves, un peu plus grandès. que les précedentes, \& qui feront également. divifées en plufieurs lots.

$$
\text { V. I. TIROIR. }
$$

447 Pareil au précedent.

$$
\begin{aligned}
& \text { VI L TI R O I R. } \\
& \text { Bivalves \& Univalves. }
\end{aligned}
$$

448 Un Fufeau bien colorié par les deux extrço inités.

449 Un grand Cour de Venus،

450 Un autre, idem, avec deux Brulées.

45 I Un Chou bien tacheté dè pourpre.

452 Un Manteau Ducal des plus-vifs en couleur.-

453 Deux beaux Toits Chinois on Pagodes, avec un Manteau Ducal.

454 Un Nautile Papiracé de mayenne grandeun 455 Un autre, idem.

456 Une groffe Fraize; un petit Coeur de Bøeuf: une Noix de mer, \& une autre Bivalve, efpece de Tricottée, affez rare.

457 Ie reftant did Tiroir: Couronnes Impéria-

- riales; Tonnes; Olives noires; Nuées; Grimaces; Culotes de Suiffe; Draps d'or; Caf ques, \&c. qui feront divifés en plufieurs lots.

$$
\text { V III. TIR OIR:- }
$$

458 Univalves Turbinites \& Bivalves, comme Oeufs, Perdrix, Figues, Radix, Equilles) Alênés, Concha Veneris, d'Ethiopie, \& autres; Cafques, Turbans, \&:c. qui feront pareillement détaillées. 
ET AUTRES CURIOSITE'S. 45

IX. TIROIR,

Rempli de Bivalves précieufes.

459 Une belle Pinne marine, avec une groffe Tuilée à tuiles plates.

460 Deux autres Tuilées d'une efpece différente

à la précedente.

46I Une Concha Veneris, avec un Coeur de Bøuf.

462 Une autre Concha Veneris, avec une autre Bivalve finguliere.

463 Une pelure d'Oignon très-délicate, avec une Moule particuliere, \& une autre Bivalve violette.

464 Une Huitre épineufe, avec deux jolies Bivalves blanches, tachetées d'un brun vif. 465 Une petite Huitre feuillée, tachetée de pourpre, \& un Cœur de Venus.

466 Une autre Huitre épineufe, crue \& attachée dans le fond d'une autre Coquille, morceaus fort particulier.

467 Deux petites Huitres épineufes de diverfe efpece, \& une petite Tricotée. Cette derniere eft rare.

468 Deux Moules particulieres, de couleur d'Agathe \& d'un bel orient, avec une autre $\mathrm{Bi}$ valye.

$469 \mathrm{U}_{\mathrm{n}}$ petit Marteau. Tout le monde connoit la rareté de cette Coquille, c'eft une des plus diftinguées parmi les Curieux.

470 Une belle Huitre épineufe, fur laquelle une autre Bivalve efpece de crête de coq, eft adhérante.

47 I Une Huitre feuillée, bien tachetée de pour-

pre. C'eft l'efpece la plus rare des Huitres ćpineufes.

472 Une autre Huitre tachetée de violet, \& adhé- 
46 HISTOIRE NATURELLE,

rante à un morceau de coral blanc.

473 Une Crête de coq, d'une efpece peu commune à pointes rondes \& feuillées.

474 Deux petites Arches de Noé coloriées : cette efpece eft rare à trouver, coloriée \& conditionnée; avec deux Moules fingulieres.

475 Une Huitre épineufe de couleur d'orange: cette couleur eft des plus rare en coquillages. $476 \mathrm{Le}$ refte du Tiroir : petites Huitres épineufes \& de belle couleur, avec d'autres Bivalves fingulieres \& parfaites, qui feront divifées en plufieurs lots.

\section{$X$. TIROIR.}

Ce Tiroir eft garwi de Bivalves auffi rares que les précedentes.

477 Un Marteau plus grand que le précedent.

478 Trois Lepas vivement tachetés, qui ferort vendus feul à feul.

479 UnEventail, autrement la Sole; parce qu'elle eft brune par-deffus, \& blanche par-deffous; el!e eft d'une grandeur au-deffus de toutes celles que j’ai poffédé, \& très-difficile à trouver de cette forme.

480 Deux Rapes \& une Pinne marine. 48I Lne Pelure d'oignon coloriće d'une moyenne grandeur.

482 Un Groupe de deux Huitres épineufes adhérantes l'une à l'autre.

483 In autre Eventail, avec un Manteau Ducal. 484 Une moyenne Crête de coq.

485 Deux Moules de couleur d'Agathe, parfaitement belles.

486 Une petite Huitre ćpineufe, \& une petite feuillće.

487 C'te autre petite Huitre adhérante z̀ un Coral blanc, \&r un petit Chou. 
ET AUTRES CURIOSITES, 47 483 Idem.

489 Deux Bivalves rares \& fingulieres, avec une Hyrondele parfaitement confervée; ce qui n'eft pas ordinaire à cette efpece.

490 Une Peiure d'oignon, autrement appellée la Selle Polonoife ou l'Ephippium, de la plus grande efpece \& du plus bel orient. C'eft une Coquille au moins auffi rare que le Marteau, $\&$ des plus renommées. Je n'en ai jamais poffedé qu'une dans le premier Coquillier que j'ai apporté de Hollande.

491 Le reftant du Tiroir en Moules, \& autres Bivalves particulieres.

\section{$X I$. T I R O $\mathrm{R}$.}

492 Bivalves \& Univalves de la petite efpece: Cadrans ou Efcaliers, Mufiques, Limas, Cornets de S. Hubert; \& autres jolies efpeces qui feront détaillées.

\section{TIROIR.}

493 Idem.

\section{TIROIR.}

494 Une Tonne tachetée, la Vis de preffoir ou le Tirefond, avec une autre grande Eguille. 495 Un beau Scorpion.

496 Deux petits Tigres de la rare couleur, une Brunette; \& une grande Olive à bandes de la belle efpece ; cette derniere n'eft pas commune.

497 Une Chicorée \& une grande Grimace.

498 Deux Damiers à bandes jaunes, un Drap d'or \& un Brocard de foye.

499. Deux'Tigres, un Oeuf \& un Cierge ou Onix; cette derniere eft bien rare \& furtout à trouver bien faine, \& je n'en ai point eu depuis le premier Coquillier. soo Une Tourterelle blanche, c'eft l'efpece la 
48 HISTOIRE NATURELLE. plus rare, avec deux groffes Olives à bandes de la belle efpece.

50I Une petite Epineufe de la rare efpece à fond \& blanc \& ćpines brunes, la Flambée \& une autre belle volute.

502 Une petite Araignée mâle, une Tonne tachetée \& canellće \& une Chicorée.

g03 le refte du Tiroir : Ourfins de diverfes efpeces, Cafques, Olives noires \& autres, Argus, Ecorchées ou Nućes, \&c.

\section{TIROIR.}

$504 \mathrm{Ce}$ Tiroir contient environ une trentaine de morceaux de mine d'argent de différentes végétations, parmi lefquelles il y en a de riches \& de fingulieres, quelques petits morceaux de mine d'or, un autre morceau de mine de diamant, plufieurs criftaux, quelques pétrifications, quelques tablettes d'encre de la Chine, le tout fera détaillé à la vente.

$\mathrm{X}$ V. ET XVI. TIROIR S.

505 Ces deux Tiroirs contiennent douze boëtes de Papillons étrangers, des plus beaux \& des mieux confervés; quelques autres. Infectes, des Champignons de diverfes efpeces, \& autres Madrepores peu communs.

\section{V I I. TIR OIR:}

Ce Tiroir eft rempli de morceaux choifis.

506 Deux grands Damiers à bandes jaunes, \& deux autres Volutes.

507 Deux Pinnes marines de diverfes efpeces; la Pintade rare, \& une autre Bivalve auffi peu commune.

508 Un beau Scorpion.

sog Une efpece de Cœur de bœuf à pointes, de couleur 
ET AUTRES CURIOSITE'S. 4,

couleur d'orange, extrêmement rare: je ne

l'ai poffedé que cette fois.

$\varsigma$ 10 Une Huitre épineufe, adhérante à un caillou. 5 II Idem.

5 I 2 Un Groupe de deux Crêtes de coq parfaites.

513 Deux groffes Olives, de la belle efpece ì bandes, avec une petite Bécaffe épineufe.

514 Un Murex couvert d'un Madrepore, morceau fingulier.

515 Une Plote de beurre, \& un Tigre de la rare couleur.

5 I 6 Un Murex raboteux ou à cloux, rare.

517 Une Feuillée parfaite, tachetée de pourpre. 518 Une belle grande Tuilée.

519 Deux petites Epineufes à fond blanc, de la rare efpece.

520 Une petite Huitre feuillće, \& une autre avec épines.

52 I Deux Cierges ou Onix.

522 Le refte du Tiroir: Ourfins de diverfes efpeces, Tigres, Lapins, Huitres, \&c.

X V I I I. TIROIR.

\section{Pareil au précedent.}

523 Une Couronne d'Ethiopie, \& trois autres

Turbinites, d'efpeces variées \& rares.

524 Un Groupe de trois Huitres épineufes : morceau fingulier \& très-confervé.

525 Un graníd Chou très-frifé.

526 Une Papiracée \& deux Tonnes, d'une efpe-

ce peu commune.

527 Un Groupe de trois grandes Crêtes de coq. 528 Une extremement grande Huitre épineufe d'une efpece rare, dont les épines font rangćes régulierement.

529 Une efpece d'Huitre feuillée jaune \& adhérante à un Madrepore. 
50 HISTOIRE NATURELIE;

530 Une autre, idem, fort finguliere.

531 Deux Nautiles de Papier, l'une à oreille, \& l'autre fans oreille.

532 Une grande Turbinite, efpece de figue, dont la bouche eft à gauche. Géneralement toutes les Coquilles ont l'ouverture de la bouche à droite; $\&$ il n'y en a que cinq ou fix efpeces, dans lefquelles cette ouverture fe trouve à gauche, \& que l'on nomme ordinairement uniques, à caufe de cette fingularité. Celleci eft une de ces efpeces $\&$ des plus rares.

533 Une Huitre d'une efpece finguliere \& trèsrare; \& une Arche de Noé.

534 Un gros Drap d'or facié; c'eft la plus belle efpece.

535 Quatre Murex d'efpeces peu communes. $\$ 36$ Le refte du Tiroir: Cafques pavés, Rubans, Grimaces, Ecorchées, grands Fufeaux, \&c.

\section{TIROIR.}

537 Mineraux, Criftaux , Bois pétrifié, \& autres pétrifications; Poiffons imprimés fur pierre \& fur ardoife, \&c.

$$
\mathrm{XX} \text {. TI ROI R. }
$$

$53^{8}$ Une Tourterelle blanche, \& une Epineufe de la rare efpece.

539 Une Pinne marine \& une Solle.

540 L'Unique; efpece de figue, dont la bouche eft à gauche, plus petite que la précedente.

\$4I Une petite Huitre épineufe, avec un Groupe de deux Crétes de coq.

542 Un petit Arrofoir ou Brandon de l'anour. C'eft un morceau des plus rares; il eft gravé dans le Suplénent du Recreatio mentis of oculi, au numero 45 . Le Pere Bonanni rapporte qu'il ne connoit aucun Auteur qui en 
ait parlé, \& qu'il ne le fçait que dans le fameux cabinet du Grand Duc de Tof cane; il eft de l'Ifle d'Amboine; il l'appelle Teftaceum Anonimum, \& paroît pancher à le mettre dans la claffe des Tubes.

543 Un petit Fufeau à côte d'une efpece beaucoup plus rare que les autres, \& coloriépar tout. Je ne l'ai poffedé que cette fors. $544 \mathrm{Un}$ autre, idem, plus grand.

545 Deux Tourterelles de la belle efpece, \& deux Olives à bandes.

\$46 Un Manteau Ducal, \& une autre Bivalve vive en couleur \& peu commune.

$\$ 47$ Une belle Tricotće.

5.48 Une Huitre épineufe crue \& adhérante dans le fond d'un des côtés d'un cœur de boeuf en Arche de Noé. Jeu de nature très-fingulier. \$49 Une Arche de Noé coloriée, \& un petit Chou.

550 Une Crête de coq à pointes, \& une autre Bivalve; toutes deux couvertes de plufieurs petits coquillages, que l'on appelle Tulipes de mer.

F5 I Efpece de Manteau Ducal pourpre, àTubercules, extrêmement rare, \& que je n'ai point encore poffedé. Il fe trouve aufi gravé au numero I2. du Suplément au Livre de Reoreatio mentis $\mathcal{F}^{\prime}$ oculi. Le Pere Bonanni. dit qu'il eft à jufte titre porté à un haut prix par les Curieux. Il croit fur les rivages du Brefil. 552 Un Concha Vencris \& un Cadran.

553 Idem:

\$54 Le refte du Tïroir: Cafques pavés, Rubans, Argus \& Bivalves fingulieres.

\section{XI. T1R $01 \mathrm{R}$.}

555 Deux Toits Chinois \& deux Brúlées. 556 Un. Chou des plus coloriés, \& de la plus E ij 


\section{HISTOIRE NATURELLE,}

groffe efpece : c'eft un des beaux qui fe puiffe trouver, aufi-bien que plufieurs autres qui font dans ce Coquillier.

557 Une Pelure d'oignon, \& deux efpeces de Noix de mer vivement colorićes.

558 Une belle Huitre épineufe.

559 Une autre, idem, d'un beau violet. 560 Une Grimace \& un Tigre, toutes deux de l'efpece rare, avec une Huitre épineufe. 561 Une autre Huitre épineufe à pointes jaunes. 562 Un Murex épineux, \& deux beaux Cœurs de bouf de diverfes efpeces.

563 Un grand Cour de Venus.

564 Un Groupe de deux Crêtes de coq très-vives en couleur.

565 Un Murex raboteux ou à clous, de la rare efpece.

566 Une groffe Tricotée.

567 Deux Figues, deux Volutes particulieres; \& deux Limas à peau de ferpent.

568 Le refte du Tiroir.

\section{TIROIR.}

569 Turbinites de diverfes efpeces, qui feront détaillées.

\section{XIII. TIROAR.}

570 Plufieurs belles Plantes Corrallhoides \& flexibles, de couleurs vives, \& rangées dans dec cahiers de papier blanc.

57 Plufieurs grandes Pierres quarrées \& tendres, fur lefquelles il y a des Plantes \& Paifages imprimés par la nature : elles forit des plus belles de ce genre, $\&$ des plus vivement coloriées.

XXIV. TIR OIR.

572 Divers Mineraux \& Madrepores. 
ET AUTRES CURIOSITE'S. 53 X X V: Ti Rо $1 \mathrm{k}$.

57; Un beau Drap d'or, de la plus groffe efpece: 574 Un très-gros Oeuf; un moyen Drap d'ox fort beau; les Nuées, \& un Ourfin ou Bouton de mer.

57, Deux très-beaux Oeufs; une Plume ou Mitre, \& une Thiare.

576 Un Geographique ;une Tonne, \& une $V$ eft is Perjica peu cominune.

577 Une belle Chicorée.

578 Cne autre, idem, avec deux Nućes ou Ecorchées.

579 Le refte du Tiroir: Couronnes d'Ethiopie; Porcelaines; Tigres ou Damiers; Scorpions femelles; Cafques cendrés, \&c.

\section{$X X V I . T \& R O 1 R$.}

\section{Coquilles d'ane plus groffe efpece.}

ऽBo Une belle Tonne, tachetée \& cannellée. $58 \mathrm{I}$ Une Veft is Perfica, \& un Murex fingulier. 582 Deux Perdrix, \& une Couronne d'Ethiopie.. 583 Deux grands Murex, d'une efpece particiliere.

584 Une Chicorée; un Turban ou Morefque, \& une Argus.

585 Le refte du Tiroir qui fera détaillé.

\section{TIRO1R.}

\section{Ce Tiroir eft plein de morceaux choifis.}

\$86 Deux grands. Tigres à bandes jaunes, \& deux Lapins.

587 Deux Bécaffes épineufes, très-vives en couleur, \& deux autres Turbinites fingulieres. 588 Une grande Tafe de Neptune, de couleur d'A gathe peu commune. 
14 HISTOIRE NATURELLE;

589 Un gros Chou, vivement tacheté.

590 Deux belles Pinnes marines, l'une à pointes, \& l'autre fans pointes.

99 I Une Tuilée de couleur jaune, \& de la groffe efpece, parfaitement confervée; c'eft un des beaux morceaux du Coquillier.

59. Unc moyenne Taffe de Neptune, \& une Couronne d'Ethiopie, brune.

5.93 Deux parfaites Brulées, de diverfes efpeces. $\$ 94$ Une grande Margaritifera, toute couverte de Tubes vermiculaires; morceau extrćmement fingulier.

$\$ 95$ Un grand Fufeau colorié, de la rare efpece. $\$ 96$ Une grande Thiare d'un beau rouge vif.

$\$ 97$ Une Plote de beurre, brillante \& vive en couleur.

9.98 Un grand Cierge ou Onix des plus blanc: cette Coquille eft difficile à trouver belle; $\&$ je n'en ai point eu depuis le premier $\mathrm{Co}$ quillier.

599 Une Geographique \& une Argus, de la plts groffe efpece; \& une Tonne peu commune.

600 Un grand Drap d'or facié, extrêmement vif en couleur.

6o I Une grande Huitre épineufẹ, adhérante à un marceau de rocher.

$6 \mathrm{O}_{2}$ Une autre, idem, \& fur laquelle il fe trouve deffus un morceau de Crête de cog adhérant.

X X VIII. TIROAR.

403 Taffes \& Secoupes, d'ancien vernis.

XXIX. TIROIR.

604 Coquilles de diverfes grandeurs \& efpeces; qui feront détaillées. 
ET AUTRES CURIOSITE'S. Ss $\mathrm{X} X \mathrm{X}$. TIROIR.

Pierres gravées en creux do en relief, montées ¿ non montées, \& Portraits émaillés.

\author{
PREM IER BOETTIER.
}

605 Une grande Agathe Onix des trois couleurs repréfentantes une fainte Face gravée ea relief, montée en or.

606 Une Iris gravée de relief des deux côtez; avec une bordure d'or.

607 Un S. Nicolas gravé fur du Jad, avec une

bordure émaillée fur or; N. S. gravé fur du Jafpe \& une tête de Chrift gravcé en creux fur une grande \& belle Sardoine.

608 Une Diane antique gravée de relieffur une Sardoine Onix à trois couleurs, avec une bordure d'or.

609 Sept Pierres de différente grandeur, Jacinthe, Jafpe, Lapis, gravees tant en relief qu'en creux.

610 Une tête de Chrift gravée de relief fur un beau Grenat fyrien, monté en or.

6 II Neuf Agathes Onix ou autres Pierres précieufes gravées tant en creux qu'en relief, dont deux font montées en argent.

6 I 2 N. S. gravé fur une Onix montée à fond \& bordure émaillée fur or \& entource de Pierres épaifies \& Rubis.

613 Une Cornaline propre pour un cachet, trèsbien gravée.

6I 4 Une autre, idem.

615 Henry IV. \& Louis XIII. gravés de relief fur deux Cornalines.

6 I 6 Une grande Cornaline gravée en creux, repréfentant quatre tétes.

617 Un Sacrifice parfaitement gravé de relief 
fur une grande Sardoine à plufieurs couleurs, avec une bordure d'or.

618 Une Diane d'Ephefe, antique, gravée aufí de relief lur Sardoine à plufieurs couleurs, avec une bordure émaillće fur or.

6x9 Deux Cornalines \& deux Jafpes gravées en creux.

620 Un combat fur un Pont, \& un fujet de métamorphofe, gravés en relief furdeux Agathes Onix montées en vermeil.

62 I Une très-belle Bacchante gravée en reliof fur une Agathe Onix.

622 Un Sacrifice bien gravé en creux fur une Agathe d'Orient avec une bordure d'or.

\section{I. B O E T T I E R. .}

623 Quinze Bagues montées en or \& gravées en creux \& en relieffur Onix, Jafpe, Emeraude, Amethifte, Cormaline, Grenat, \&c. parmi lefquelles il y en a beaucoup d'antiques très-bien gravées, \& qui feront détaillées.

I I I. B OETTIER:

624 Quinze autres Bagues, idem. .

I V. B OETTIER.

625 Plufieurs Bagues \& Cachets gravés fur Cornaline \& autres Pierres montées en argent \& tombac, avec quelques autres de compofition, faites la plupart d'après plufieurs belles antiques, qui feront détaillées à la.vente.

$$
\text { V. BOETTIER. }
$$

626 Sept Agathes œillées, montées en or, qui ferons vendues en détail. 
PETIT Coffre a DIVERS TIROIRS.

627 Dans lepremier \&le deuxićme Tiroir;foixante morceaux gravés tant en creux qu'en relief fur Cornaline Onix , Jafpe, Agathe Onix, Lapis, Prime d'Emeraude, Aigue marine, Amethifte \& Cornaline, dont la plûpart font de vieille roche; toutes Pierres antiques non montées \& qui feront divifées en plufieurs lots.

628 Dans le troifiéme Tiroir; les Portraits de N. S. \&r de la Vierge très-bien émaillés fur or des deux côtez, avec une forte bordure aufî érnaillée fur or \& des plus achevées.

622 Le ?utrtait te ivi. Ki igauit Peintre du Roi, auff parfaitement peint en émail.

630 Sir autres beaux Portraits tant d'hommes que de femmes, émaillés fur or \& fur cuivre, quui feront détaillés.

631 Treize graveures de différente grandeur fur Jafpe, Amethifte, Cornaline, Lapis \& Agathe Onix, toutes antiques qui feront partatagées en divers lots.

632 Dans le quatriéme Tiroir; Une grande Onix fans être gravée.

633 Vingt-deux grandes Pierres gravćes tant en creux qu'en relief: Sardoine, Lapis, lafpe, A gathe Onix, \&c. toutes antiques qui ferontdétaillées.

Autre petit Coffre a nivers Tiroirs.

634 Un Portrait de Louis XIV. en émail, entouré de vermeil.

635 Une Sardoine taillée en fexagone,répréfentant Hercule porte bœuf, très bien gravée en creux, avec une bordure émaillée fur or, \& unse autre Sardoine ovale gravée aufi en creux \& repréfentant Moïfe qui reçoit les tables de 
S8 HISTOIRE NATUREILE, la Loi; on a profité dans cette Pierre de l'utrvantage des couleurs.

636 Soixante - douze P ierres de compofition ảe petite \& moyenne grandeur, faites d'après plufieurs belles antiques.

637 Quarante autres grandes, idem, dont la plûpart font d'après les Pierres du Cabinet du Roi.

\section{A UTRE PETIT COFFRE.}

638 Soixante-fix petites diverfes Pierres gravées en creux \& en relief, propres pour petites bagues \& cachets de montre,parmi lefquelles il y en a de fort jolies, \& qui feront divifćes en plufeurs lois.

639 Les trois petits Coffres qui renferment les Pierres cy deffus énoncées.

640 Les cinq Boettiers cy-deffus.

64I Une Agathe Onix antique gravée de relief, repréfentant Apollon \& Marfias, montée en

642 Deux autres, idem, aufi montées en or. XXXI. TIROIR.

643 Univalves Turbinites qui feront divifes en pinfieurs lots.

XXXII. 'TIROIR.

644 Boëtes, Taffes \& Secoupes d'ancien vernis. X X XIII, TIR OIR.

645 Coquilles de la groffe efpece: Couronnas d'Ethiopie, Cafques, Turbans, Chicorces \& Madrepores très particuliers qui feront détaillés:

XXXIV. TIROIR.

646 Une efpece de grand Champignon renver- 
FT AUTRES CURIOSITE'S. S,

fe, morceau des plus finguliers.

$6_{47}$ Un autre Madrepore fair en boule, aufi fingulier que le précédent.

648 Le refe du Tiroir; Coquilles de la groffe efpece, \& Madrepores diff̈̈rens.

$$
\text { XXX V. TIROI R. }
$$

649 Plufieurs Figures Hollandoifes très finies en cire, \& proprement liabillées.

$$
\text { XXXVI. TIROIR. }
$$

Coquilles de la groffe efpece.

650 Une grande Araignée mâle.

651 Un Murex fingulier, une Araignée femelle \& une belle Trompe marine.

652 Le Murex raboteux, rare \& vivement tachété de noir: il eft des plus gros que l'on puilie trouver de cette efpece.

653 Refte du Tiroir: groffes Caicorćes, Couronnes d'Ethiopie, \&c.

Sur les Tublettes qui font au defus ó à

$$
\text { coié du Coquillier. }
$$

Animaux finguliers dans desphioles: Papillons, Madrepores, groffes Coquilles, Ouvrages ex cire, animaux or Figures de Porcelaine $\mathcal{S}$ de terre ancieniue, des plus fingulieres, \& autres Curiofitez.

.654 Tro is grands Serpens : le Serpent à fonnette très-rare : un Serpent des Indes Orientales nommé l'Aveugle : un autre grand Serpent de $\mathrm{I}^{\mathrm{A}}$ Amérique : un autre Serpent des Indes avec un Soldat ou Bernard l'Hermite : un grand Cameleon : une autre efpece de Serpent des Indes ; le beau Lezard ou Legouan de Surinam : un Serpent de l'IAle de Jamai-. 

que : un Lievre de l'Amćrique : deux Plantes particulieres : une magnifique Tarentule avec une Grenouille noire : le poiffon appellé l'Efpadon : le Pareffeux avec un petit Serpent : le Cheval marin avec un beau Serpent bandé : trois Serpens de Surinam : un Poiffon fingulier \& un petit Polype : le Porc-épic avec un Serpent : une très-belle Chenille de Surinam, avec le Serpent appellé le fourd ou le double marcheur : un Lezard de P'A merique à longue queuc̈: l'Eguille \& un autre Poiffon: deux autres Poiffons finguliers :deux très-beaux Serpens : un Serpent de Malabar avec la Malfaifante ou Mille pates : un Serpent de Surinam avec un Soldat: le Mechant de l'Amérique : le Poiffon volant : un Serpent \& un Soldat : le Poiffon bandé,avec un Scorpion : une Mille pates avec une Chenille de Curfo: deux différens Serpens : le Colibri ou l'Oifeau mouche : autre efpece de Poiffon bandé : un Serpent \& une Chenille finguliere : trois Lezards d'Hollande : un Crocodile fortant de l'œuf : deux petits Polypes: le Poifion Quadrangulaire ou Diable de mer: la Chenille couronnée de Surinam avec un Scorpion : un petit infecte fingulier : Le tout faifant quarante Phioles, qui feront vendues en un feul article s'il fe trouve des Encheriffeurs qui le demandent ainfi; finon on les divifera en plufieurs lots.

655 Seize Phioles remplies d'Animaux pareils aux précedens, qui fe font trouvés doubles, \& que l'on diftribuera en plufieurs lots. 656 L'Oifeau de Paradis fans pates.

657 Deux autres Oifeaux de Paradis, l'un fans pates, \& l'autre avec pates.

On eft revenu de l'erreur où l'on étoit au fujet de cet Oifeau: on avoit tâché de faire croire 

Agates Arborisées ou Dendri

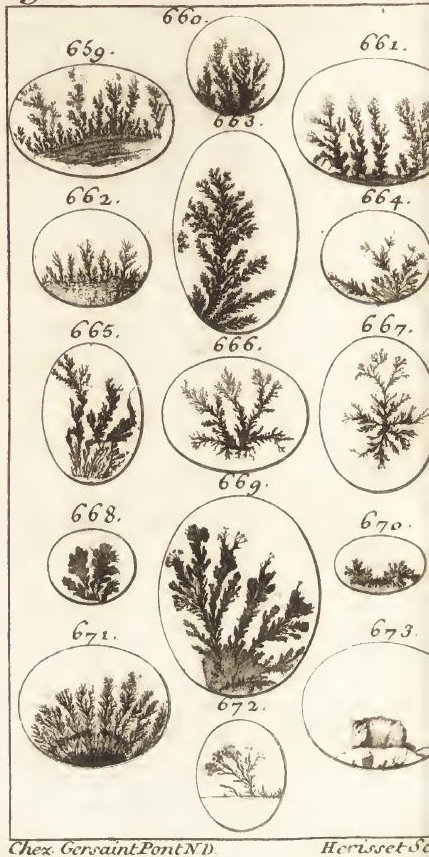




\section{ET AUTRES CURIOSITE'S. 6I}

croire que cet animal ne vivoit que de l'air dans lequel il pafioit entierement fes jours fans fe repofer, n'ayant point de pates, \& qu'il mouroit aufi-tót qu'il approchoit dela la terre ; cette erreur ćtoit entretenue par une pecite adreffe de ceux qui apportoient de ces Oifeaux dans d'autres Pays, pour s'en procurer une vente plus favorable a caufe de cetri te fingularité inaginaire, . \& de la difficulté qu'il paroiffoit y avoir à les trouver : ils leur otoient, à cet effet, fubtilement les pates, afin d'entretenir les Curieux dans cette erreur; ce qui fait qu'il eft extrèmement rare d'en trouver avec les pates, parce qu'ils n'en ont jamais apporté fans les tronquer auparavant.

658 Deux autres Oifeaux de Paradis, l'un fans pates \& l'autre avec pates.

$\{659\}$ Quinze Agathes arborifées, repréfen$\{673\}$ tant des Payfages, des Plantes, \&c. dont on peut voir les copies dans la Planche qui eft cy-à-côté. Ces Pierres feront vendues $n^{\circ}$. à n '. de la façon qu'elles font notées fur cette Planche.

674 Seize autres Pierres, idem.

675 Environ une cinquantaine de Madrepores, $\&$ autres Plantes marines, tant grandes que petites, des plus rares \& des mieux confervées, montées fur des pieds,parmi lefquelles il s'en trouve d'uniques : elles feront détaillées au gré des encheriffeurs.

676 Nombre de morceaux de Porcelaine \& Terre ancienne du Japon : Animaux, Pagodes, Hermites, Theieres, Sujets, Figures fingulieres \& de goût \&c.

677 Plufieurs Coquilles de la grofte efpece : entr'autres quatre grandes Trompes marines des 
6 HISTOIRE NATURELLE. plus belles.

678 Une Boëte remplie de Foffilles, Coquitles: \& petites Plantes, \&c.

679 Dix Boëtes de Papillons très-beaux \& autres Infectes.

680 Autres Papillons mis fous verres.

68I Plufieurs tablettes d'encre de la Chine.

682 Deux Figures en cire repréfentant un Vieux \& une Vieille : ouvrage très-fini \& très-naturel.

683 Plufieurs autres petites Figures, auffi en cire, répréfentant des habillemens différens de Paifans, Matelots \& Artifans de la Hollande, tant en hommes qu'en femmes, habillées très-proprement, \& finies avec beaucoup d'art \& de naturel.

684 Deux petites Pyramides d'ivoire extrêmement délicates, chef d'œuvre de patience.

685 Un grand plat d'albâtre de vingt-un pouces de diamettre proprement fculpté, avec fon étui : cette piece eft fort curieufe.

686 Une Boëre qui comprend cent quatrevingtdouze petites cafes remplies de différens mineraux.

687 Une grande Chambre obfcure. $6 r 8$ Un petit Bronze très-fini.

\section{Addenda aux Eftumpes.}

689 Plufieurs Eftampes de différens Maîtres Flamands, comme Goltzius, Saerdam, Rimbramt, Sadeler, \&cc, qui feront détaillées. ' 690 Les Infectes de l'Europe \& de Surinam par Mademoifelle Merian, deux volumes in-folio carta maxima, reliés en veau fäuve. 69I Deux volumes en blanc, idem. 692 Les impoftures innocentes, par Bernard $\mathrm{P}:-$ cardin-folio. 
ET AUTRES CURIOSITE'S. 63

693 Le mêne, grand papier.

694 Le volume des Coquilles de Rumphius, in.folio.

695 Le Pluvinel, in-folio.

696 Les titres, vignettes \& culs de lampe pour les Panégyriques. \& Difcours à la louange de Louis XIV. par S. le Clerc.

697 Les deux catalogues d'Eftampes de l'Abbé de Maroles, le grand \& le petit : on fçait l'extrême difficulté à trouver ce petit catalogue, dont il n'en a été tirć qu'un trèspetit nombre d'exemplaires.

698 Plufieurs Eftampes modernes montées en verre blanc \& bordures brunes, qui feront détaillées.

699 Deux petits volumes : fcavoir, un livre d'emblêmes, \& une vie de la Vierge.

700 Foachimi de Sandrart Academia nobilifimi Artis liztoria, où il fe trouve une fuite con-fidérable de Portraits de Peintres de toutes leś Ecoles. Ce volume peut fervir de fuite au $n^{\circ}$. I de cette vente.

Z 0 I Fefte publique faite àAnvers en I 594 à l'avénement du Prince Erneft Archiduc d'Autriche au Gouvernement des Pays bas, avec les Tournois faits à Stutgard en 1617 , à l'occafion du baptême d'Ulric, \& du mariage du Duc de Wirtemberg. 2. vol.

702 Un volume d'Oifeaux, \& un volume de fleurs.

703 Defcription des Côtes de Portugal par Teyxeyra Géographe du Roi de Portugal : les

- cartes y font deffinées de fa main.

704 Nombre de Porte-feuilles \& Boëtes trèspropres \& convenables pour ferrer des EItampes.

705 Un Rocher métallique renfermé dans une phiole: morceau très-rare \& de prix, dant 
64 HISTOIRE NATURELLE. les vrais Curieux connoitront le prix en l'examinant

Outre les Coquillages cy-deffus énoncés, il s'en trouve une collection autant nombreufe que j'ai pû la faire, \& où chaque efpece ne fe trouve qu'une feule foi , à moins qu'il n'y ait quelque diřerence fenfible. Cette collection comprend environ quatre cent foixante pieces, \& eft des mieux choifies pour les couleurs \& la perfection : je la vendrai à l'amiable, fi quelque Curieux fe préfente pour l'acheter.

Plufieurs Tableaux des meilleurs Maitres Flamands \& Hollandois, comme Teniers, Berghem, Vauvremens, Metzu, Therbuch, Bachuyfen, Bartholomé, un petit Claude le Lorrain, \&c. que je vendrai pareillement à l'amiable.

Fin da Catalogue.

Pour contenter les gouts différens, $j$ entre. mêler ai les articles pendant le cours de la vente, afin que closcun puiffe trouver occafion de fe fatisfaire tous les jours en quelque cbofe dans le genre de fa curiofité.

On verra reginer à cette vente la droiture o la bonne foi que l'on a toujours reconnia dans les précédentes; ce qui me fait efperer que les Anateurs y viendront avec li nich.ne confiunce 



$$
\text { ILAp96-013241 }
$$





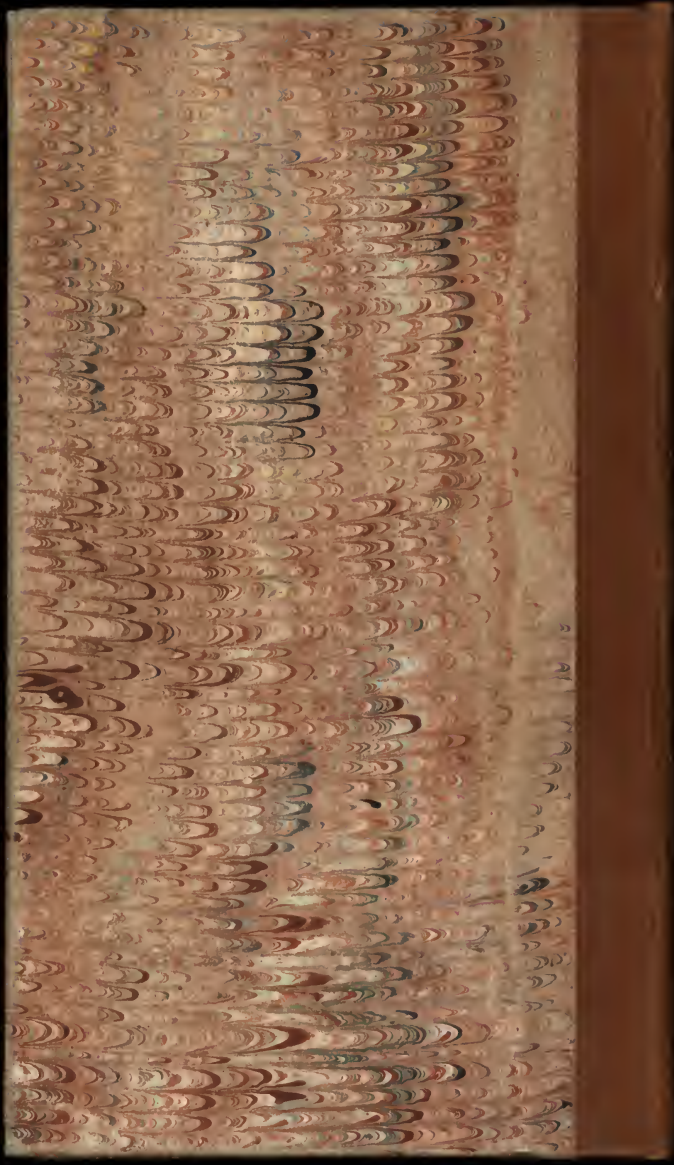




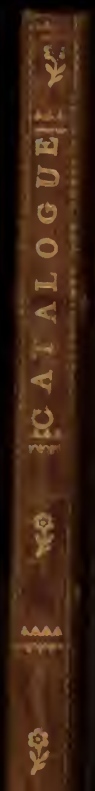

way

8
5 